\title{
Barotropic vortex pairs on a rotating sphere
}

\author{
By MARK T. DIBATTISTA AND LORENZO M. POLVANI \\ Program in Applied Mathematics and Department of Applied Physics, Columbia University, \\ New York, NY 10027, USA
}

(Received 21 February 1996 and in revised form 15 October 1997)

Using a barotropic model in spherical geometry, we construct new solutions for steadily travelling vortex pairs and study their stability properties. We consider pairs composed of both point and finite-area vortices, and we represent the rotating background with a set of zonal strips of uniform vorticity. After constructing the solution for a single point-vortex pair, we embed it in a rotating background, and determine the equilibrium configurations that travel at constant speed without changing shape. For equilibrium solutions, we find that the stability depends on the relative strength (which may be positive or negative) of the vortex pair to the rotating background: eastward-travelling pairs are always stable, while westward-travelling pairs are unstable when their speeds approach that of the linear Rossby-Haurwitz waves. This finding also applies (with minor differences) to the case when the vortices are of finite area; in that case we find that, in addition to the point-vortex-like instabilities, the rotating background excites some finite-area instabilities for vortex pairs that would otherwise be stable. As for practical applications to blocking events, for which the slow westward pairs are relevant, our results indicate that free barotropic solutions are highly unstable, and thus suggest that forcing mechanisms must play an important role in maintaining atmospheric blocking events.

\section{Introduction}

Vortex pairs, which consist of two oppositely signed regions of vorticity travelling together as a single entity, are important coherent structures of fluid dynamics, and appear in a wide variety of physical domains. Of specific interest for geophysical applications is the existence and stability of such pairs embedded in a rotating background flow with a vorticity gradient, which gives rise to the well-known $\beta$ effect. In this paper we present new vortex-pair solutions on the sphere obtained by considering simplified distributions of vorticity chosen specifically to shed some light on the interaction between the vortex pair and rotating background flow.

A broad range of vortex-pair solutions are known, from the simple modulated point-vortices on a $\beta$-plane of Zabusky \& McWilliams (1982) to the sophisticated modon solutions on the sphere of Verkley $(1984,1987)$. However, a systematic exploration of the full range of behaviour exhibited by vortex pairs and a unified explanation of sources for the various instabilities is still lacking.

The simplest studies of point-vortex pairs in rotating fluid have noted the fundamental difference in behaviour between eastward- and westward-travelling pairs. Both Hobson (1991) and Matsuoka \& Nozaki (1992) noted that the former are stable with respect to small perturbations, whereas the latter exhibit figure-eight-type large-amplitude oscillations. These models, however, suffer from the limitation that 
while the vortex-pair motion is influenced by the rotating background, the latter is unaffected by the motion of the vortex pair.

A more complex model, the 'modon' solution of Stern (1975) and Larichev \& Reznik (1976) for continuous vortex pairs on the $\beta$-plane, is capable of more subtle behaviour and has received much attention (Flierl et al. 1980; McWilliams et al. 1981; Sutyrin et al. 1994). McWilliams (1980) has applied it to the phenomenon of atmospheric blocking, and Tribbia (1984) has extended it to the spherical geometry. The early studies seemed promising in this regard; however, few of these examined the evolution of perturbed modons in order to determine their stability properties. In fact, later studies have concluded that the modon solution is unstable (Verkley 1984, 1987; Nycander 1992) in ways reminiscent of point-vortex pairs. Sutyrin et al. (1994) furthermore showed that vortex pairs may not survive when embedded in strong background flows (they did not, however, perform a complete parametric study of the possible regimes of behaviour). One aim of this study is to determine whether the instability of the modon solution is an exceptional or a generic property of vortex pairs in rotating flows.

We do this by constructing new solutions whose simplicity allows us to identify the mechanisms of the instability. These solutions are obtained from a constructive approach: we start by considering each one of the constituent elements in isolation the vortex pair first, the rotating background next - and then we couple them together and determine their mutual influences. For simplicity, the rotating background flow is approximated by strips of uniform vorticity, while the vortex pair is formed either of point vortices or uniform patches of oppositely signed vorticity. The advantage of our approach is that we are able to isolate which instabilities are due to the vortex pair, which are due to rotating background flow and which are due to their coupling.

The paper proceeds from the simplest to the most complex vorticity configurations as follows. After reviewing some basic facts about barotropic dynamics on the sphere, we construct point-vortex pairs in $\S 3$ and the discretized uniformly rotating background in $\S 4$. We then consider in $\S 5$ the simplest coupled system: a pointvortex pair and two nearly-zonal contours. We determine both linear and nonlinear equilibrium solutions and then investigate their stability. In $\S 6$, while maintaining the simplicity of point-vortices, we generalize the results to a multi-contour background rotation, and we demonstrate how the two-contour system is able to capture the main qualitative features of the problem. Finally in $\S 7$, we consider finite-area vortex patches, and show how the desingularization of the point vortices leads a further instability mechanism related to the finite size of the vortices. A discussion concludes this study in $\S 8$.

\section{Barotropic dynamics on the sphere}

We use throughout this study the simplest possible fluid model of geophysical relevance, the so-called 'barotropic model', in which the fluid is incompressible, inviscid, of constant density and, in our case, lies on the surface of a unit sphere $S$. The dynamics of such a fluid is dictated by the material conservation of vorticity $\omega$ :

$$
\frac{\mathrm{D} \omega}{\mathrm{D} t}=0,
$$

where $\omega \equiv \boldsymbol{x} \cdot(\nabla \times \boldsymbol{u}), \boldsymbol{u}$ is the two-dimensional velocity of the flow and $\boldsymbol{x}$ designates the position on the sphere. Though the fluid covers a two-dimensional spherical surface, it 
is analytically and computationally simpler to express all vector and scalar quantities in terms of the three-dimensional Cartesian coordinates $(x, y, z)$, as opposed to the more common latitude and longitude angular coordinates (Dritschel 1989; Polvani \& Dritschel 1993, hereinafter PD93).

Incompressibility requires that $\nabla \cdot \boldsymbol{u}=0$ and implies the existence a streamfunction $\psi(\boldsymbol{x})$ of the form $\boldsymbol{u} \equiv \boldsymbol{x} \times \nabla \psi$. This yields the usual relationship between twodimensional vorticity and streamfunction $\omega=\nabla^{2} \psi$. Inversion of this equation results in the well-known expression

$$
\psi(\boldsymbol{x})=\iint_{S} G\left(\boldsymbol{x}, \boldsymbol{x}^{\prime}\right) \omega(\boldsymbol{x}) \mathrm{d} A^{\prime},
$$

where the Green function $G\left(\boldsymbol{x}, \boldsymbol{x}^{\prime}\right)$ for the sphere is identical in form to the corresponding Green function in the plane (Kimura and Okamoto 1987; Dritschel 1989),

$$
G\left(x, x^{\prime}\right)=-\frac{1}{4 \pi} \ln \left|x-x^{\prime}\right|^{2} .
$$

Using the inversion expression (2) the velocity field can be written, after some manipulation, in the form:

$$
\boldsymbol{u}(\boldsymbol{x})=-\iint_{S} G\left(\boldsymbol{x}, \boldsymbol{x}^{\prime}\right)\left[\left(\boldsymbol{x}^{\prime} \times \nabla^{\prime}\right) \omega\left(\boldsymbol{x}^{\prime}\right)\right] \mathrm{d} A^{\prime},
$$

showing that, along with (1), the vorticity distribution $\omega$ entirely determines the evolution of the flow.

Finally, it is worth recalling that Gauss' theorem provides a strong constraint on which vorticity distributions may exist on the sphere, since

$$
\iint_{S} \omega\left(\boldsymbol{x}^{\prime}\right) \mathrm{d} A^{\prime}=\iint_{S} \nabla^{2} \psi \mathrm{d} A^{\prime}=\iint_{S} \nabla \cdot \nabla \psi \mathrm{d} A^{\prime}=0,
$$

and the last equality follows from the fact that the sphere has no boundaries. Thus only vorticity fields that integrate to zero are allowed on the sphere.

\section{Point-vortex pairs in a non-rotating background}

We start with the simplest vorticity configuration of relevance, a pair of point vortices on the sphere with strengths $\Gamma_{+}$and $\Gamma_{-}$of opposite sign, located at $\boldsymbol{x}_{+}$ and $\boldsymbol{x}_{-}$, respectively and lying on the same longitude. As illustrated in figure 1, two parameters suffice to describe such a pair geometrically: the chord distance $d$ between the point vortices and the latitude $\lambda$ of the midpoint between the two vortices.

Solutions for the special case $\Gamma_{+}=-\Gamma_{-}$are given in Lamb (1932), who showed that such a pair travels along a great circle. Since in following sections we will be considering solid-body rotation of the fluid about the $z$-axis, we choose here, without loss of generality, to have the vortex-pair rotate about the $z$-axis as well, which naturally coincides with the pole. To do this, we extend the solutions given by Lamb, and consider pairs where $\Gamma_{+} \boldsymbol{x}_{+}+\Gamma_{-} \boldsymbol{x}_{-}=G \hat{\boldsymbol{k}}(\hat{\boldsymbol{k}}$ being the unit vector in the $z$-direction) so that the pair travels around the pole at constant latitude $\lambda$. The ratio $\Gamma_{+} / \Gamma_{-}$that results from the above expression defines the latitude $\lambda$. Furthermore, and again without loss of generality, we may select $G=1$; this sets the centre of vorticity to unit magnitude, which is equivalent to choosing a timescale, and uniquely determines the values of $\Gamma_{+}$and $\Gamma_{-}$. Hence, once $\lambda$ and $d$ are chosen, the point-vortex pair is completely defined. 


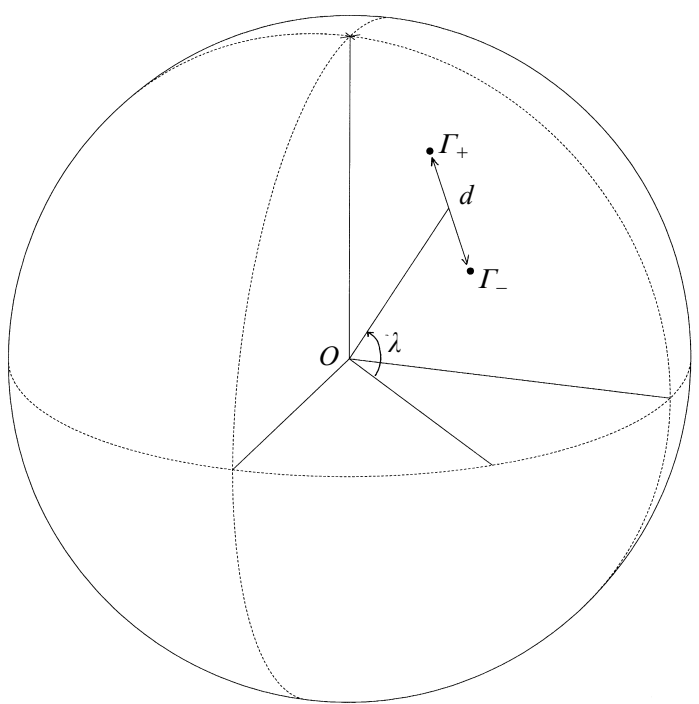

FiguRE 1. A point-vortex pair on the sphere, and the associated parameters. The dots indicate the positions of the point vortices.

Notice that not all values of $d$ and $\lambda$ yield vortex pairs. Since the vortex strengths are of opposite sign, the vortices must be placed 'on the same side of the sphere' in order to go around the pole. This implies the existence of a maximum latitude $\lambda_{\max }=\left(1-(d / 2)^{2}\right)^{1 / 2}$ for any given separation distance $d$, otherwise one vortex will be forced beyond the pole.

In figure 2 we draw the streamlines for two pairs with $d=0.2$ : an equatorial pair $\left(\lambda=0^{\circ}\right)$ and a midlatitude pair $\left(\lambda=45^{\circ}\right)$. It is clear that the streamline patterns of these spherical pairs bear close resemblance to their planar counterparts (see, for instance, Batchelor 1967). In the inertial frame the streamlines are circular for the case $\lambda=0^{\circ}$ (figure $2 a$ ), as noted by Lamb, while for the more general case the streamlines bend with no simple description of the shape (figure $2 b$ ). In the corotating frame (figures $2 c$ and $2 d$ ), i.e. the frame of reference where the vortex pair is stationary, the patterns shows the existence of regions of trapped fluid travelling with the pair, and of two 'critical' points where the separatrices crosst; notice also that, except in the immediate vicinity of the pair, the corotating streamlines are nearly zonal.

It should be easy to see that point-vortex pairs are stable for all parameter values, in the sense that small initial perturbations in their initial positions simply redefine the axis and speed of rotation, while the two vortices still travel together as a single entity.

\section{Contour discretization of the solid-body rotation}

In this section - and throughout this paper - we consider a spherical barotropic flow in solid-body rotation whose vorticity is a linear function of $z=\sin \vartheta$ (where $\vartheta$ is the latitude), going from 1 at the north pole to -1 at the south pole; with this choice, the angular velocity of the flow is $\frac{1}{2}$. Solid-body rotation thus implies a uniform gradient of vorticity, and this is well known to support vorticity waves,

$\dagger$ It can be shown that the two critical points coalesce for $d=\sqrt{ } 3$ and disappear for large $d$. The trapped-fluid regions then encompass the entire spherical surface. 

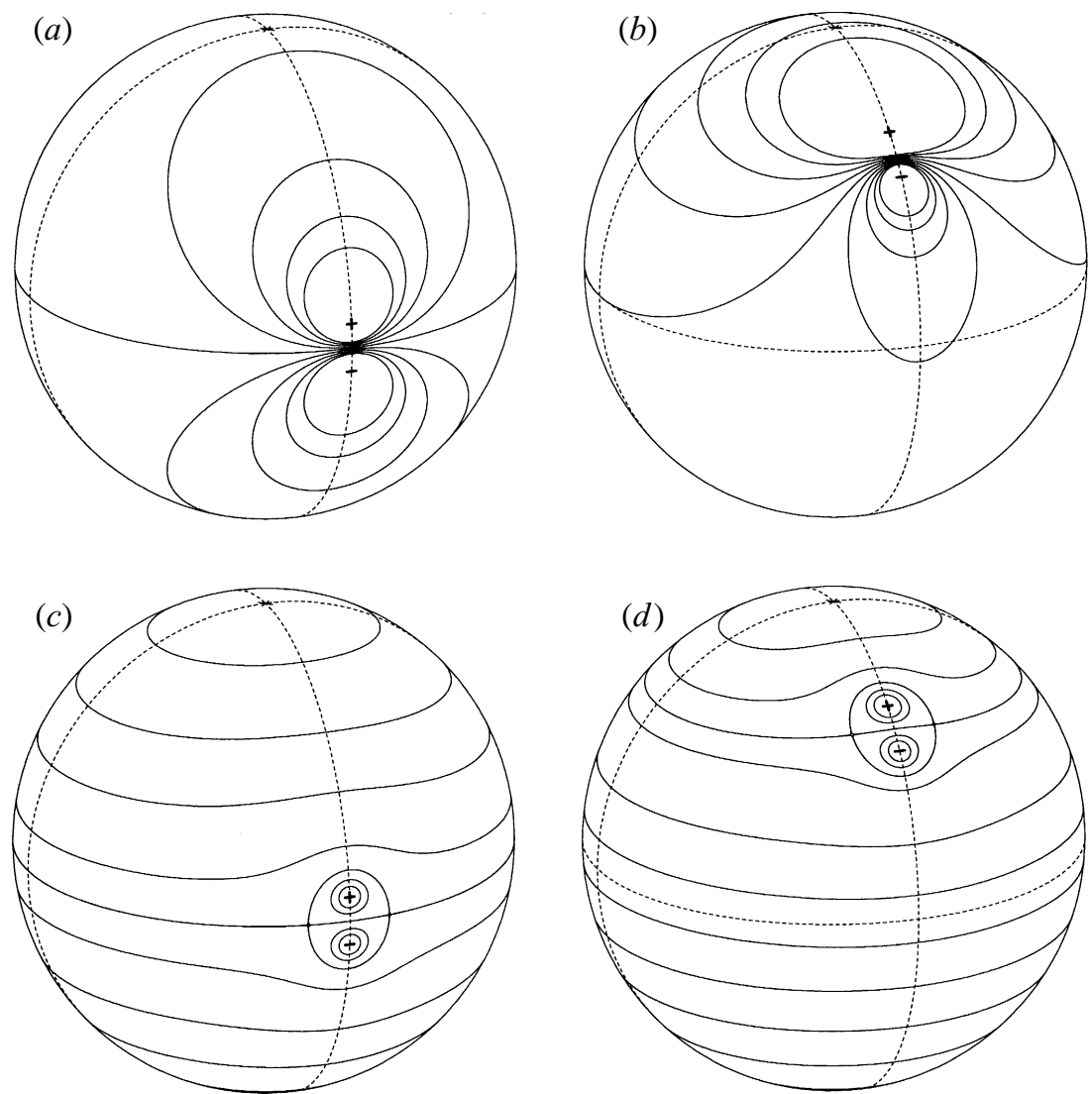

FIGURE 2. The streamlines associated with spherical point-vortex pairs in the inertial $(a)$ and $(b)$ and corotating $(c)$ and $(d)$ frames, for an equatorial $\left(\lambda=0^{\circ}\right)$ case $(a)$ and $(c)$ and a midlatitude $\left(\lambda=45^{\circ}\right)$ case $(b)$ and $(d)$. The distance between the point vortices is $d=0.2$. The positions of the positive and negative vortices are marked ' + ' and '-', respectively. The streamlines are not uniformly spaced in value, and are chosen to illustrate the flow pattern associated with the vortex pairs.

which on the sphere are called Rossby-Haurwitz waves (Haurwitz 1940). The aim of this section is to represent the solid-body rotation in such a way that its coupling to the point-vortex motion can be treated with a maximum of simplicity.

To this end, we discretize the above linear vorticity distribution by considering $N+1$ zonal strips of uniform vorticity separated by $N$ contours. For simplicity, we take the strips to be of equal area, so that the $N$ contours separating them are located at positions

$$
z_{0 k}=1-\frac{2 k}{N+1},
$$

where $k$ labels the contours, from 1 to $N$. The value of vorticity in each strip is chosen to be the average of the linear profile of solid-body rotation within that strip. This implies that the vorticity jump $\tilde{\omega}_{k}$ across the $k$ th contour is given by

$$
\tilde{\omega}_{k}=\frac{1}{2}\left(z_{0(k-1)}-z_{0(k+1)}\right),
$$

where we define $z_{00} \equiv 1$ and $z_{0(N+1)} \equiv-1$. For piecewise-uniform vorticity distributions, the expression (4) for the velocity reduces to a sum of simpler one-dimensional 
integrals around the $N$ contours $\mathscr{C}_{k}$,

$$
\boldsymbol{u}(\boldsymbol{x})=-\sum_{k=1}^{N} \tilde{\omega}_{k} \oint_{\mathscr{C}_{k}} G\left(\boldsymbol{x}, \boldsymbol{x}_{k}\right) \mathrm{d} \boldsymbol{x}_{k} .
$$

For a derivation of this identity, and many other details on Contour Dynamics, see Dritschel (1989). With the vorticity jumps selected according to (7), the angular velocity computed from (8) is exactly equal to $\frac{1}{2}$ at each contour location of the discretized system.

In order to evaluate the effectiveness of this strip-wise discretization, we have tested how well it is able to capture the most important behaviour of a fluid in solid-body rotation, namely the Rossby-Haurwitz waves. Following Dritschel \& Polvani (1992, hereinafter DP92), we perturb each zonal contour $\mathscr{C}_{k}$ by a small amount $\zeta_{k}(\phi, t)$, where $\phi$ is the longitude, so that the $k$ th contour is located at

$$
z_{k}(\phi, t)=z_{0 k}+\zeta_{k}(\phi, t) .
$$

Substitution of this into the $z$-component of the velocity expression (8) yields, upon linearization,

$$
\frac{\mathrm{d} \zeta_{k}}{\mathrm{~d} t}=\frac{\partial \zeta_{k}}{\partial t}+\frac{1}{2} \frac{\partial \zeta_{k}}{\partial \phi}=-\sum_{j=1}^{N} \frac{1}{2 \pi N} \int_{0}^{2 \pi}\left|\boldsymbol{x}_{0 j}(\alpha)-\boldsymbol{x}_{0 k}(\phi)\right|^{2} \frac{\partial \zeta_{j}}{\partial \alpha} \mathrm{d} \alpha
$$

which is identical to equation (8) of DP92, with $\Omega_{0 k}=\frac{1}{2}$ and $\widetilde{\omega}_{k}=2 / N$. Letting

$$
\zeta_{k}=\hat{\zeta}_{k} \mathrm{e}^{\mathrm{i}(m \phi-\sigma t)}+\text { c.c., }
$$

where $m$ is the azimuthal wavenumber of the perturbation, yields the eigenvalue problem

$$
[\boldsymbol{A}-\sigma \boldsymbol{I}] \hat{\boldsymbol{\zeta}}=0,
$$

where $\sigma$ is the frequency of the eigenmode, and $\hat{\zeta}$ is a vector of length $N$ whose components are the amplitudes $\hat{\zeta}_{k}$. $\boldsymbol{A}$ is an $N \times N$ matrix with components

$$
A_{j k}=-\frac{2}{N} F_{m}\left(z_{0 j}, z_{0 k}\right)+\delta_{j k} \frac{m}{2},
$$

and, as in DP92,

$$
F_{m}\left(z_{a}, z_{b}\right)=\left(\frac{1-z_{>}}{1+z_{>}} \frac{1-z_{<}}{1+z_{<}}\right)^{m / 2},
$$

where $z_{>}=\max \left(z_{a}, z_{b}\right)$ and $z_{<}=\min \left(z_{a}, z_{b}\right)$.

For a given number of contours $N$, equation (12) yields $N$ meridional eigenmodes for each azimuthal wavenumber $m$. Each of these is the discretized equivalent of the corresponding continuous Rossby-Haurwitz wave whose structure is the spherical harmonic $Y_{m}^{n}$, where $n=m+p$; the number $p(p=0, \ldots, N-1)$ represents the meridional wavenumber of each mode.

In figure 3 , we show the phase speeds $\sigma / m$ for the lowest eight values of the total wavenumber $n$, versus the number of contours $N$. The dotted lines show the values $\frac{1}{2}-1 /(n(n+1))$, which are the phase speeds of the continuous Rossby-Haurwitz waves (Haurwitz 1940). It is clear that the phase speeds of the discretized Rossby-Haurwitz waves quickly approach the continuous values as $N$ increases; it would seem that as 


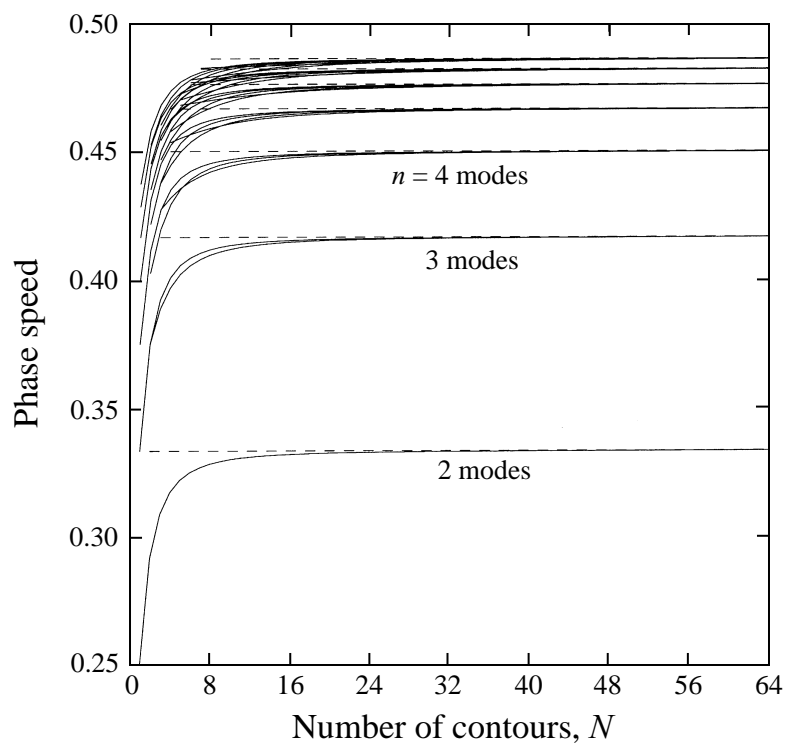

FIGURE 3. Phase speeds of linear waves propagating on a background in solid-body rotation discretized by $N$ contours. The dashed lines show the phase speeds of the continuous Rossby-Haurwitz waves; they also correspond to the discretized $m=1$ waves (which happen to propagate at the exact phase speed of the continuous Rossby-Haurwitz waves). For the discrete waves, as for the continuous waves, the phase speed accumulate at $\frac{1}{2}$ as $n \rightarrow \infty$.

few as eight contours give a fair approximation for the large-scale modes. Notice that as $n$ becomes large, the continuous phase speeds accumulate at $\frac{1}{2}$ (as do the discrete phase speeds); this is the angular velocity of the solid-body rotation.

Beyond the linear approximation, one could compute exact discrete nonlinear analogues of Rossby-Haurwitz waves; one avenue would be to extend the algorithm of PD93 to many contours. Since this is not the main purpose of the present study, we limit ourselves here to showing how an approximation to a large-amplitude discrete Rossby-Haurwitz wave maintains its shape over many rotations.

To accomplish this, we place the $N$ contours along the non-zonal streamlines of the continuous Rossby-Haurwitz wave such that all strips of vorticity enclose an equal area; the vorticity jump is therefore the same across each contour. This is equivalent to using expression (7) where the value of $z_{0 k}$ refers to the average latitude of the $k$ th contour. Although such a construction is not an exact nonlinear discrete Rossby-Hauwitz wave, it behaves very much like one.

Figure 4 shows the evolution of a 16-contour approximation to a Rossby-Haurwitz wave with $n=5$ and $m=3$, whose continuous analogue is stable according to Baines (1976). After six wave periods there is little noticeable change in shape. As expected for such waves, the motion is retrograde with respect to the solid-body rotation. We have found similar robust behaviour for many discrete versions of Rossby-Haurwitz waves, with a variety of wavenumbers $(n, m)$ and number of contours $N$.

From such experiments we conclude that a strip-wise discretization of solid-body rotation, as described above, retains the most important dynamical features of the continuous problem. Moreover, a relatively small number of contours suffice to do this. 

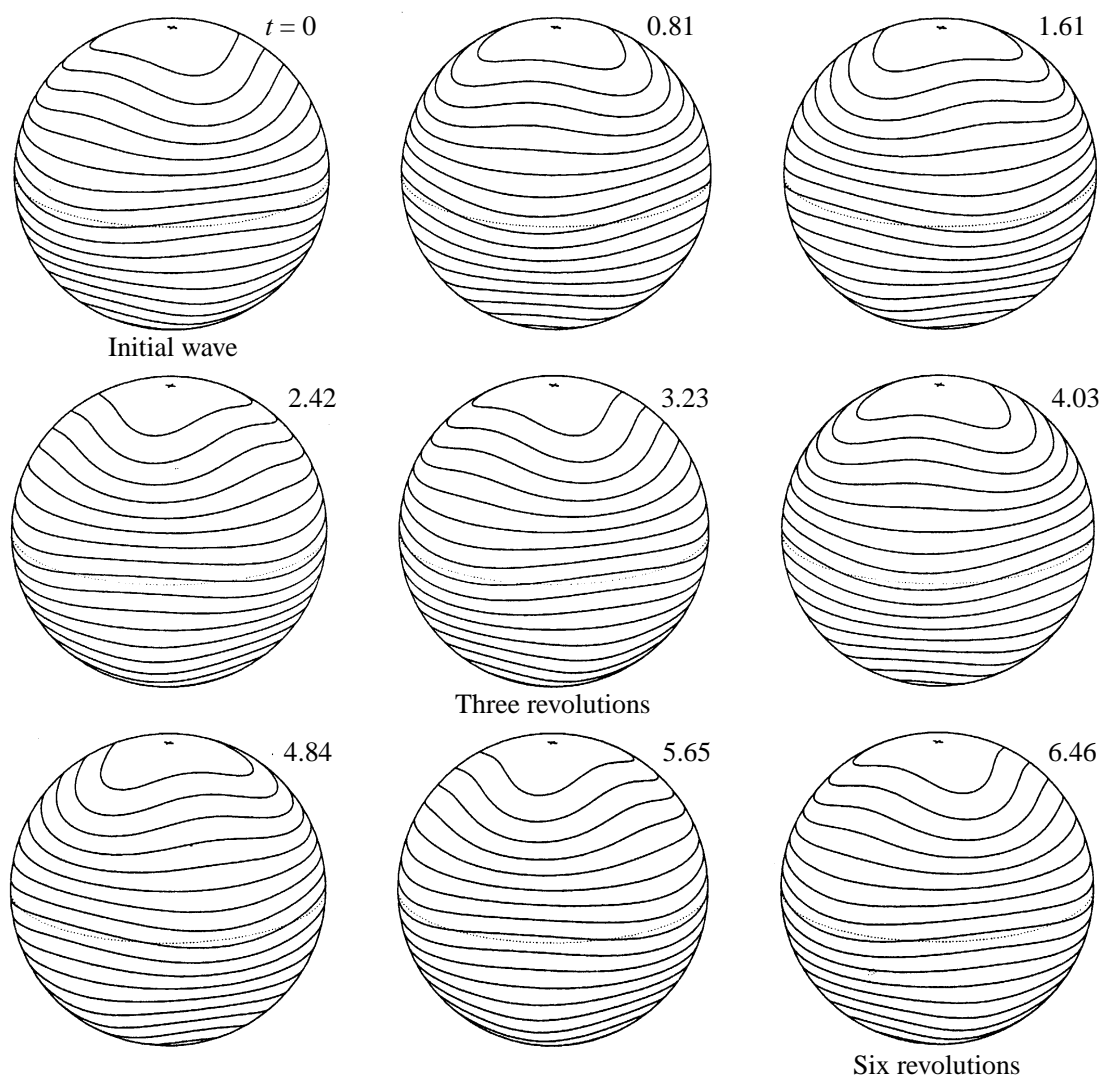

FiguRE 4. An example of an approximate discrete Rossby-Haurwitz wave with $n=5$ and $m=3$. Six periods of the wave are shown in the inertial frame. The time $t$ is in 'days', measured by the revolution time of the background that is in solid-body rotation.

\section{Point-vortex pairs in a two-contour background}

Given that relatively few contours can capture the general behaviour of Rossbywave propagation, we now consider a point-vortex pair in a background solid-body rotation with only two contours, as illustrated in figure 5. As we will show, this simplest configuration is sufficient to capture most of the qualitative behaviour of the interaction between the point-vortex pairs and the solid-body rotation.

Although extremely idealized, the configuration of figure 5 is defined by several parameters. In addition to the distance $d$ between the two point vortices in the pair and the latitude $\lambda$ of the pair, two more geometrical parameters appear to be required for the two contours representing the solid-body rotation, namely the location $z_{01}$ and $z_{02}$ of the two contours. However, in order for the pair to rotate uniformly about the pole, the contours must induce the same angular velocity on both vortices, and this implies that only one parameter may be specified. We choose this parameter to be the vertical distance $D$ between the two contours; given $D \equiv z_{01}-z_{02}$, the values of $z_{01}$ and $z_{02}$ are thus uniquely determined.

In addition to these geometrical parameters, the vortex strengths $\Gamma_{+}$and $\Gamma_{-}$as well as the vorticity jumps across the contours $\tilde{\omega}_{1}$ and $\tilde{\omega}_{2}$ must be specified. The latter two are chosen using equation (7), which sets to unity the centre of vorticity associated with the solid-body rotation. As in $\S 3$, we must require that the pair travel uniformly 


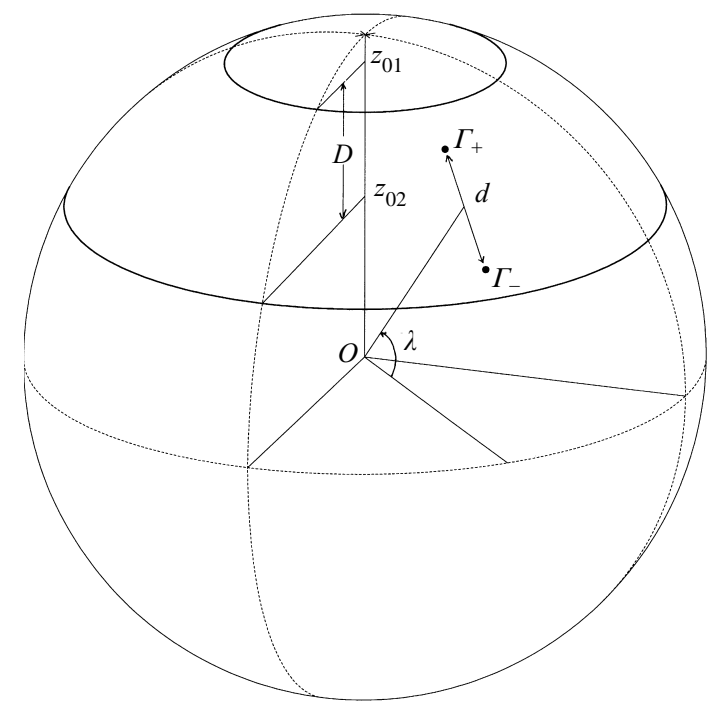

FIGURE 5. Geometric parameters for a point-vortex pair with two background contours.

about the pole, which requires $\Gamma_{+} \boldsymbol{x}_{+}+\Gamma_{-} \boldsymbol{x}_{-}=G \hat{\boldsymbol{k}}$. Unlike $\S 3$, however, we cannot set $G=1$ here without loss of generality.

In fact, $G$ is the key parameter of this and the remaining sections. It measures the relative strengths of the point-vortex pair to the solid-body rotation. Positive $G$ corresponds to eastward-travelling vortex pairs, negative $G$ to westward-travelling pairs. In the limits $G \rightarrow \pm \infty$ the solid-body rotation is very weak in comparison to the strength of the pair, and the solutions of $\S 3$ are recovered. The interesting behaviour occurs for $|G| \leqslant 1$, when the vortex pair is comparable to or weaker than the rotating background flow.

In order to limit the amount of computational work, we have opted not to systematically explore all the possible values of the four parameters $\lambda, d, D$ and $G$, but to focus only on a few representative cases. To this end, we have considered only two values of $\lambda$ chosen to yield the qualitative latitudinal dependence of the results: an equatorial case $\left(\lambda=0^{\circ}\right)$ and a midlatitude case $\left(\lambda=45^{\circ}\right)$. For configurations of practical interest, changes in the parameters $d$ and $D$ do not qualitatively affect the results; hence, we have not methodically explored all values of these parameters. The parameter $G$, on the contrary, is crucial in determining both the shape of the equilibrium solutions for vortex pairs and their stability; we have thus explored many values of this parameter, as detailed in what follows.

\subsection{Linear two-contour equilibrium solutions}

We first determine the linear equilibrium solutions for a steadily-translating vortex pair surrounded by two contours described by the above parameters. Since the vortex locations are set a priori, the solution consists in determining the linear perturbations induced by the vortex pair upon the contours. This is easily done with a slight modification of the expressions used in $\S 4$.

The non-zonal component of the contour locations $\zeta_{k}(\phi, t)$, see (9), obey an equation 
similar to (10) except for two extra terms due to the point vortices:

$$
\begin{aligned}
\frac{\mathrm{d} \zeta_{k}}{\mathrm{~d} t}=\frac{\partial \zeta_{k}}{\partial t}+\frac{1}{2} \frac{\partial \zeta_{k}}{\partial \phi}= & -\sum_{j=1}^{2} \frac{1}{4 \pi} \int_{0}^{2 \pi}\left|\boldsymbol{x}_{0 j}(\alpha)-\boldsymbol{x}_{0 k}(\phi)\right|^{2} \frac{\partial \zeta_{j}}{\partial \alpha} \mathrm{d} \alpha \\
& +\frac{\Gamma_{+}}{2 \pi}\left(\frac{\boldsymbol{x}_{+} \times \boldsymbol{x}_{0 k}}{\left|\boldsymbol{x}_{+}-\boldsymbol{x}_{0 k}\right|^{2}}\right) \cdot \hat{\boldsymbol{k}}+\frac{\Gamma_{-}}{2 \pi}\left(\frac{\boldsymbol{x}_{-} \times \boldsymbol{x}_{0 k}}{\left|\boldsymbol{x}_{-}-\boldsymbol{x}_{0 k}\right|^{2}}\right) \cdot \hat{\boldsymbol{k}}
\end{aligned}
$$

where $\boldsymbol{x}_{+}$and $\boldsymbol{x}_{-}$are the location of the point vortices, $\boldsymbol{x}_{0 k}$ is the position along the $k$ th contour in the absence of point vortices, and $k=1,2$. The linear equation is asymptotically valid in the limit of weak point vortices, which corresponds to $G \ll 1$. We then expand $\zeta_{k}(\phi, t)$ in azimuthal components

$$
\zeta_{k}=\hat{\zeta}_{k} \mathrm{e}^{\mathrm{i}\left(m \phi-\sigma_{G} t\right)}+\text { c.c. }
$$

where the angular velocity $\sigma_{G}$ is determined uniquely by the choice of the parameters $d, \lambda, D$ and $G$, and is the sum of the angular velocity of the vortex pair plus the angular velocity induced by the contours on the vortex pair. Substitution of this into (14) yields the following linear equation for each mode $m$,

$$
\left[\boldsymbol{A}-\sigma_{G} \boldsymbol{l}\right] \hat{\boldsymbol{\zeta}}=\hat{\gamma},
$$

where $\hat{\gamma}$ is a vector of length $N$ whose components $\hat{\gamma}_{k}$ represent the meridional velocity induced by the two point vortices on the $k$ th contour, projected on the mode with azimuthal wavenumber $m$. Contrasting this equation with equation (12) it is clear that the point vortices act as forcing terms on the contours.

The inhomogeneous matrix equation (16) can be solved provided the angular velocity $\sigma_{G}$ of the solution does not match the phase speed of one of the freely propagating eigenmodes of $\boldsymbol{A}$. The eigenmodes of $\boldsymbol{A}$ are simply discretized Rossby waves, as described in $\S 4$, and their phase speeds are retrograde with respect to the solid-body rotation, which is equal to $\frac{1}{2}$ with our scaling. Hence, there is a clear asymmetry between eastward- and westward-travelling solutions; for $\sigma_{G}>\frac{1}{2}$ solutions always exist, while for $\sigma_{G}<\frac{1}{2}$ there is a countably infinite set of values for which $\hat{\zeta} \rightarrow \infty$, two for each $m$ in this simplest two-contour case. These resonances are located in a band corresponding to slow westward solutions, which we refer to as the 'forbidden region', and accumulate at the angular velocity $\frac{1}{2}$ of the solid-body rotation.

In figures 6 and 7, we show the amplitudes of the linear solutions for two cases, each with representative values $d=0.2$ and $D=0.5$. The first case, for $\lambda=0^{\circ}$, corresponds to an equatorial pair, for which the undisturbed contours are symmetrically located across the equator. This implies that $\hat{\zeta}_{1}=-\hat{\zeta}_{2} \equiv \hat{\zeta}_{0}$ for all $m$. We show $\hat{\zeta}_{0}$ as a function of $G$ for several values of $m$ in figure 6. Notice that each curve diverges at a single value of $G$, at which point $\sigma_{G}$ is identical to the phase speed of the $m$ th varicose mode of the matrix $\boldsymbol{A}$. The sinuous modes do not cause resonances owing to the symmetry in the right-hand side of (16). The dark vertical lines delimit the forbidden region where resonances occur.

In figure 7, the amplitudes are illustrated for the second case, a midlatitude vortex pair with $\lambda=45^{\circ}$. Since here $\hat{\zeta}_{1} \neq-\hat{\zeta}_{2}$ they need to be shown separately, $\hat{\zeta}_{1}$ in figure $7(a)$ and $\hat{\zeta}_{2}$ in figure 7(b). For this midlatitude case, each curve diverges at two distinct values of $G$ as $\sigma_{G}$ resonates with both the sinuous and the varicose eigenmodes of $A$ for each $m$. Again, the dark vertical lines delimit the forbidden region.

Notice that the $m=1$ mode is special. Although figures $7(a)$ and $7(b)$ show a 


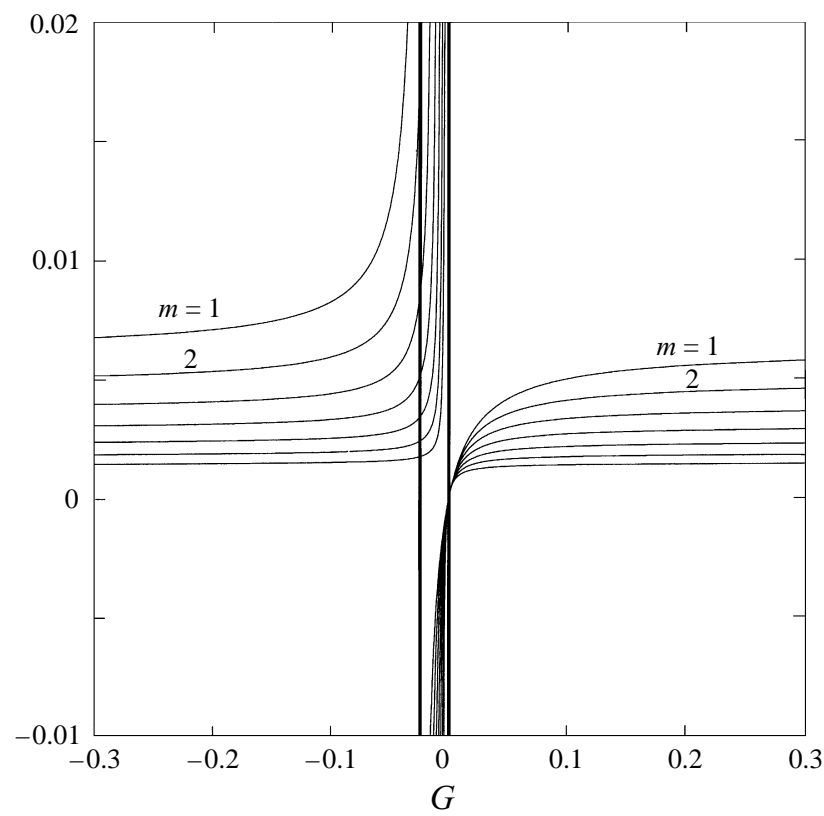

FIGURE 6 . The linear amplitudes induced by an equatorial point-vortex pair upon the symmetric two-contour background as a function of the parameter $G$, which measures the ratio of the vortex-strength to the solid-body rotation represented by the two contours. Each curve corresponds to a different azimuthal wavenumber $m$; the curves for $1 \leqslant m \leqslant 7$ are shown. The dark vertical lines demarcate the forbidden region where resonances occur.

(a)

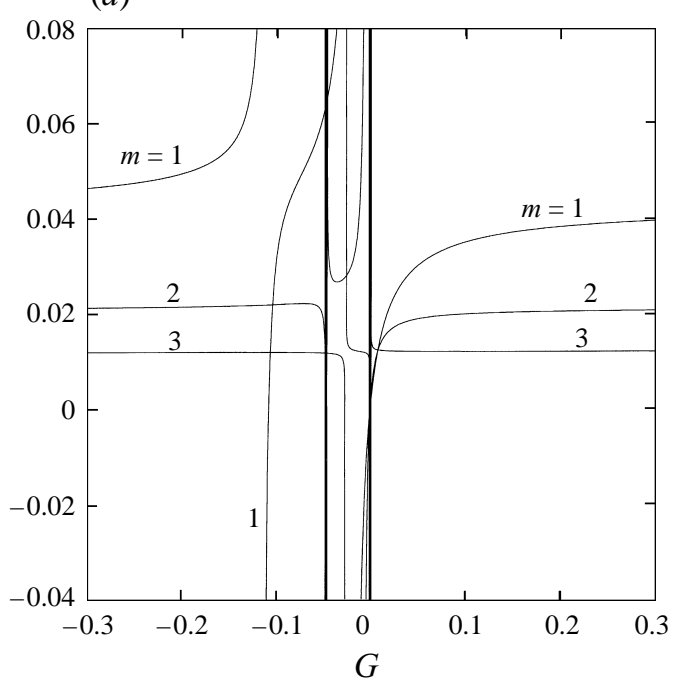

(b)

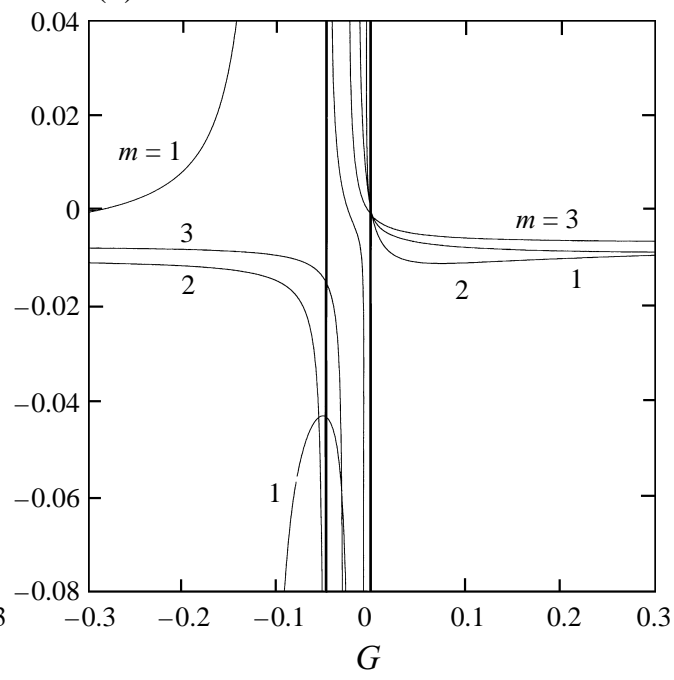

Figure 7. (a) The linear amplitudes induced by the vortex pair on the upper contour, for the midlatitude case $\lambda=45^{\circ}$. For the sake of clarity only the curves for $m=1,2,3$ are shown. The dark vertical lines demarcate the forbidden region. The left-most resonance associated with the $m=1$ mode is spurious, as explained in the text. $(b)$ As $(a)$, but for the lower contour. 
resonance near $G=-1.2$ for the $m=1$ mode, this resonance is spurious, since the $m=1$ sinuous mode moves the angular momentum of the solid-body rotation off the pole and is thus not allowed. Hence, the outermost resonance, defining the edge of the forbidden region, is given by the $m=2$ mode. The signature of the azimuthal wavenumber of the eigenmode that defines the left-most boundary of the forbidden region, which is different in the two cases above, will become apparent in the character of the nonlinear solutions.

\subsection{Nonlinear two-contour equilibrium solutions}

We now extend the above linear results and determine equilibrium solutions for a pair of point vortices and two contours where the deviations of the contours from latitude circles are not assumed to be infinitesimal. For this we have extended the algorithm of PD93 to allow for the presence of point vortices. Given values of the parameters $d$, $\lambda, D$ and $G$ which describe a specific vorticity distribution similar in topology to the one shown in figure 5, the algorithm determines both the positions of the contours and the angular velocity $\sigma_{G}$ such that the system (comprised of two point vortices and two contours) rotates at constant $\sigma_{G}$ about the pole without change in shape.

The details of the algorithm are quite involved, and can be found elsewhere (DiBattista 1997). Suffice it to say the algorithm, a modification of that used in PD93, starting from some initial condition, iteratively tries to make the contour positions coincide with the streamlines of the flow, thus producing a solution which does not change in shape. To account for the significant contour deviations from zonality in the presence of the point-vortex pair, we must modify the way in which vorticity jumps are assigned to the contours in a way that is consistent with their representing a flow in solid-body rotation. Hence we generalize definition (7) with

$$
\tilde{\omega}_{k}=\frac{A_{k-1}-A_{k}}{4 \pi},
$$

where $A_{k-1}$ and $A_{k}$ are the areas of the vorticity strips bounding the $k$ th contour. This definition reduces to (7) in the case of zonal contours.

Figures $8(a)$ and $8(b)$ show a range of nonlinear equilibria for both the equatorial and midlatitude vortex pairs, respectively, and for values of the vortex-strength ratio $G=-1.0,-0.1,-0.01,1.0$. These four values of $G$ span the range of behaviours exhibited by point-vortex pairs, which will be discussed subsequently. Notice that for the nonlinear equilibria, the deviations of the contours do not greatly differ from one another except for the equilibria corresponding to $G=-0.01$, for which the linear solution is placed squarely in the forbidden region. The deviation of the equatorial equilibria for $G=-0.01$ has a pronounced $m=1$ character (figure $8 a$ ), while the deviations of the midlatitude equilibria for the same $G$ have a definite $m=2$ character (figure $8 b$ ), both of which match the slowest linear eigenmodes excited by the point-vortex pair.

Figures $9(a)$ and $9(b)$ show the speeds of the equatorial and midlatitude nonlinear equilibria, respectively; the dashed line represents the phase speeds of the linear solutions while the squares, circles and crosses locate a few of the nonlinear solutions we have found. The most striking feature of the diagrams are the deviations of the nonlinear speeds when approaching the forbidden regions: the nonlinear phase speeds slow down and ultimately turn back (figure $9 b$ ), never crossing into the forbidden region. The maximum nonlinear phase speed for negative $G$ is greater for equatorial point-vortex pairs than for midlatitude pairs, since the forbidden region for the 

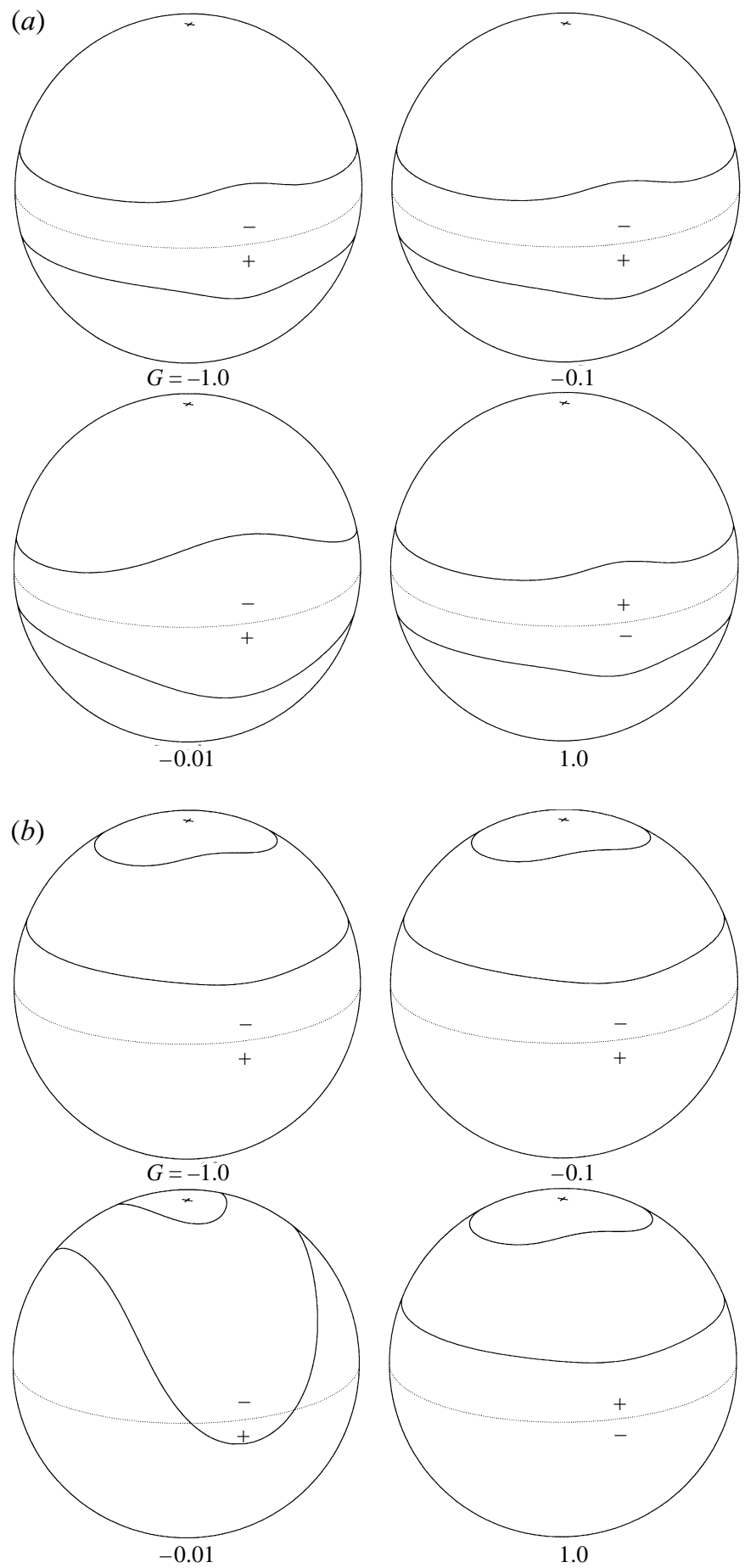

FigURE 8. (a) Nonlinear equilibrium solutions for a point-vortex pair and a two-contour background for the equatorial case for $G=-1.0,-0.1,-0.01$ and 1.0. These values of $G$ span the full range of stability behaviour. Notice that the maximum contour deformation occurs for small negative values of $G$, corresponding to weak westward-travelling pairs, and it projects mostly on the varicose $m=1$ mode. (b) As in $(a)$, but for the midlatitude case. The contour deformation at $G=-0.01$ projects mostly on the sinuous $m=2$ mode, as suggested by linear theory. 


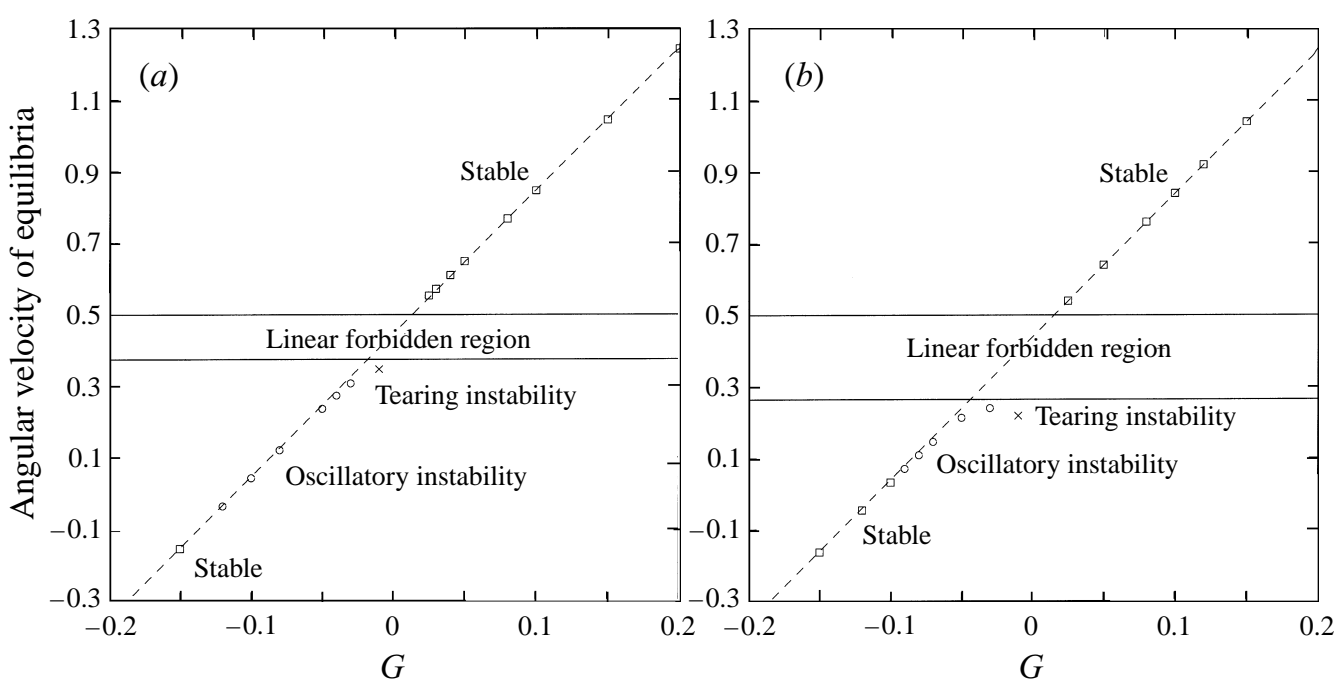

FIGURE 9. (a) The angular velocities and stability behaviour of the nonlinear two-contour point-vortex equilibrium solutions (illustrated in figure $8 a$ ). The different symbols mark the different stability behaviour, as explained in the text. The dashed lines show the phase speeds of linear solutions. (b) As in $(a)$, but for the midlatitude case.

midlatitude pair is larger. The different symbols refer to the stability of the solutions, as discussed next.

\subsection{Stability of the nonlinear two-contour equilibrium solutions}

Instead of carrying out a complicated eigenvalue stability calculation of the above vortex-pair nonlinear equilibrium solutions (along the lines, for instance, of Dritschel 1985), we have opted for the simpler method of numerically integrating the evolution of initially perturbed equilibria. We use the Dritschel's (1989) Contour Surgery algorithm, modified to account for the presence of point vortices, to integrate our initial-value problems. This approach, while perhaps not as precise as a full linear analysis, yields not only the stability boundaries of the problem but also gives insight into the nonlinear evolution of the disturbed vortex pairs.

In order to establish the sensitivity of the results to the initial conditions, we have tried several different types of perturbations: gently shifting the pair latitudinally from its equilibrium position, slightly distorting the contours in front or behind the vortex pair, and mildly tilting the vortex pair in the meridional direction. We have found that the latter method is the one that generates the clearest instabilities. Hence, in the following figures, we have perturbed the equilibrium solutions by rotating the point vortices $2.5^{\circ}$ about their common centre. Stable equilibria preserve their shape for dozens of rotations, whereas unstable equilibria generally evolve very quickly, often within a rotation time.

The key result is the following: point-vortex pairs in a rotating background composed of two contours exhibit only three distinct types of stability behaviour on the sphere. They may be stable, they may exhibit what we term an 'oscillatory' instability, in the sense that the point vortices oscillate around the equilibrium latitude while remaining together as a pair, or they be may torn apart by what we term a 'tearing' instability, in which case the point vortices are separated by the background flow. Not only does this general result apply to the two-contour solutions in this section, but it 

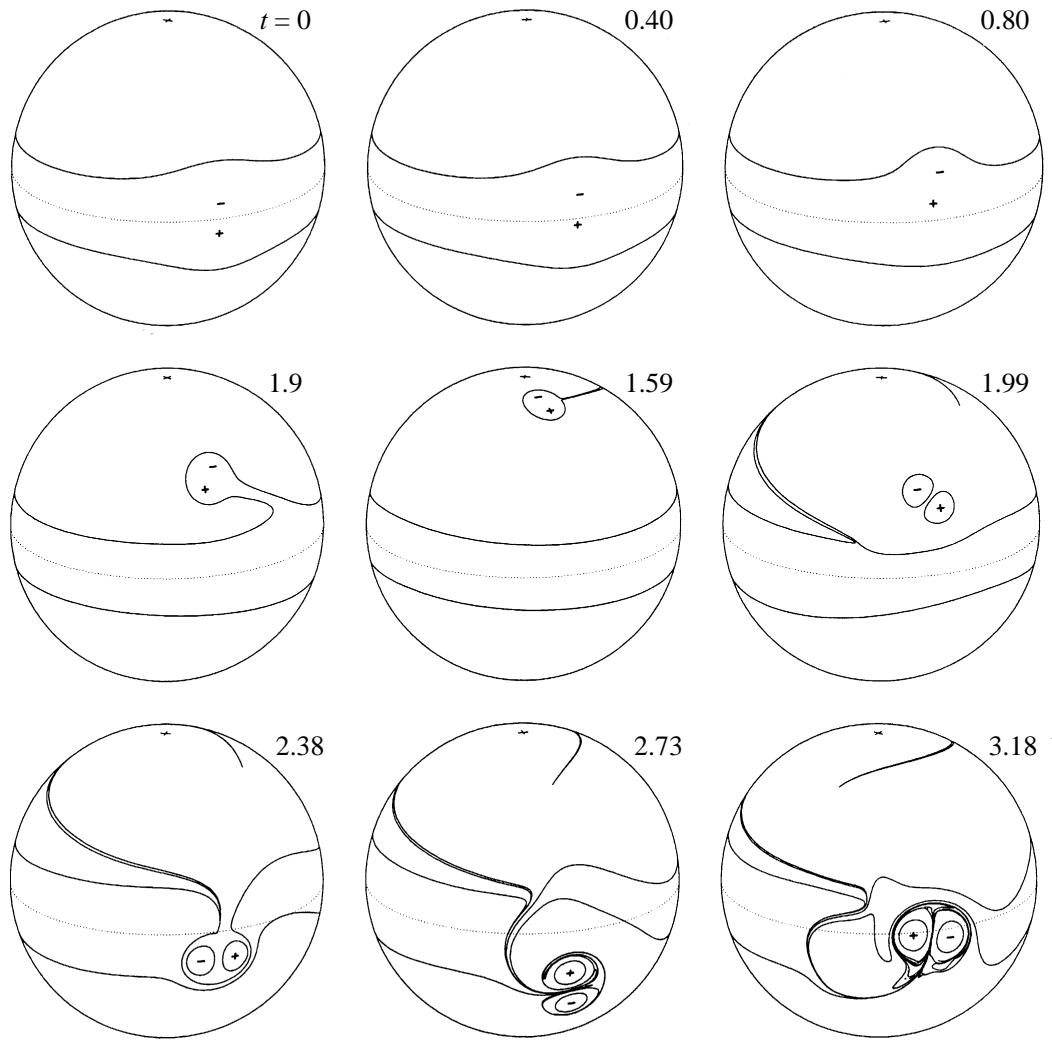

FIGURE 10. The evolution of an unstable 'oscillating' pair with $G=-0.05$. The pair is initially tilted by $2.5^{\circ}$ with respect to zonal direction. Time is measured in days, and the plots are rotated so that the pair always appears at the same longitude.

holds for the cases of multi-contour background and of finite-area vortices described in later sections.

Stable point-vortex pairs, which occupy both ends of the nonlinear phase-speed curves, are marked with squares in figures $9(a)$ and $9(b)$. All eastward-travelling pairs $(G>0)$ were found to be stable. For westward-travelling pairs, on the contrary, there is a threshold beyond which the pairs are sufficiently weak with respect to the background and become unstable; in the equatorial case $\left(\lambda=0^{\circ}\right)$ this occurs around $G=-0.15$, while for midlatitude pairs $\left(\lambda=45^{\circ}\right)$ this occurs around $G=-0.1$. Perturbed stable pairs wobble about the initial latitude in response to the perturbation, but this excursion in latitude does not grow with time, and the background contours never develop any discernible wave activity.

Unstable oscillating point-vortex pairs were found in the interval $-0.1<G<-0.02$ for the equatorial case, and $-0.15<G<-0.02$ for the midlatitude case, and are marked with circles in figures $9(a)$ and $9(b)$. The latitudinal excursions of these perturbed unstable pairs grow in amplitude, and for sufficiently small $G$ assume figure-eight-like trajectories. An example of this behaviour is shown in figure 10, for an equatorial case with $G=-0.05$. Notice that the pair remains a coherent structure as it wanders about the sphere.

Unstable tearing point-vortex pairs were found in the small interval $-0.02<$ $G<0.0$ for both equatorial and midlatitude cases, and are marked with crosses in 

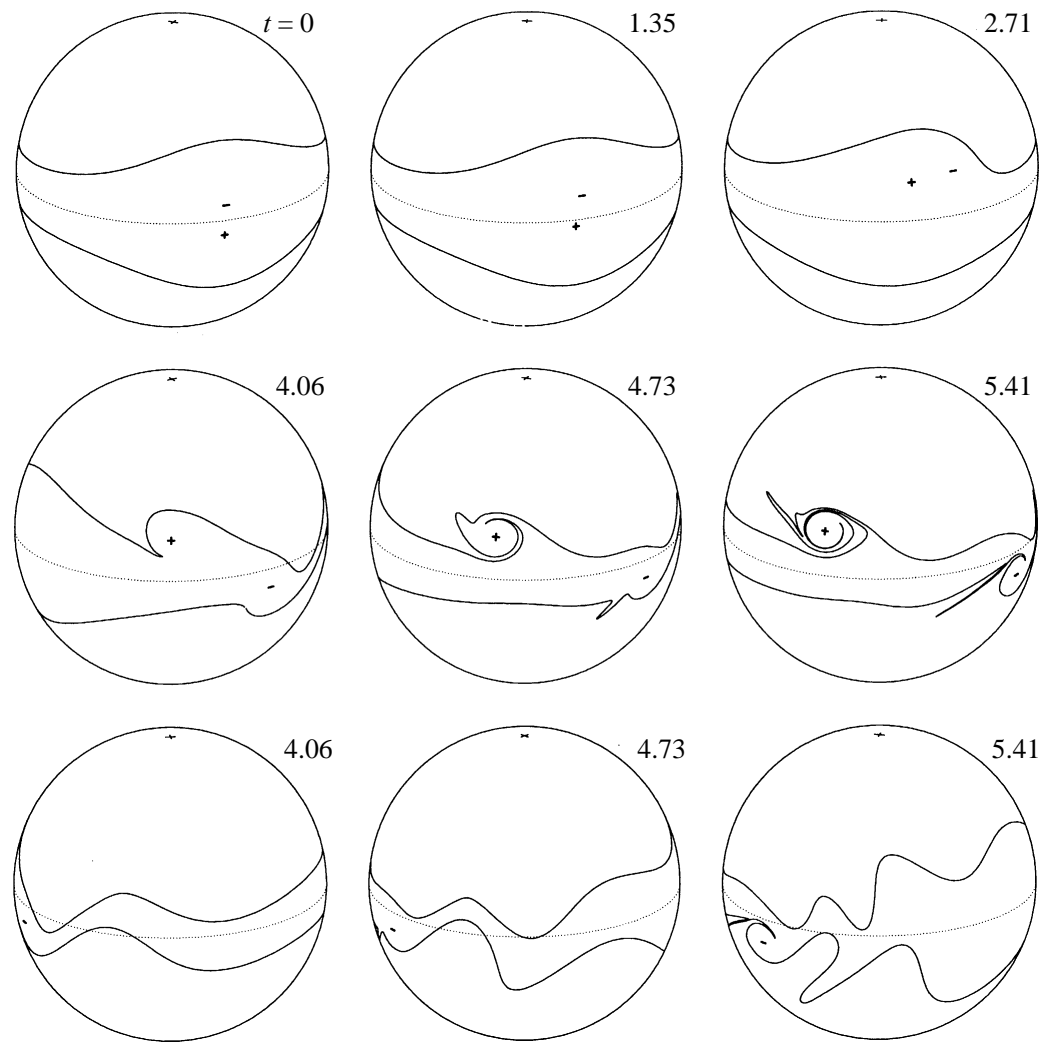

FiguRE 11. As in figure 10, but for an unstable 'tearing' pair with $G=-0.01$. In order to show the wave activity excited on the background contours as the pair is torn apart, the bottom row shows the same three time frames as the middle row, rotated westward by $90^{\circ}$.

figures $9(a)$ and $9(b)$. The evolution of these pairs is characterized by a slow increase in the distance between the two vortices, as the pair is torn apart by the much stronger background contours. An example of this behaviour is given in figure 11, for the case $G=-0.01$. It is interesting to note that, as the vortices separate, a large amount of wave activity is excited on the background contours (the last row of figure 11 shows the same time frames as the middle row rotated by a quarter turn) indicating a strong energy exchange between the pair and the background flow.

\section{Point-vortex pairs in a multi-contour background}

We now increase the complexity of the problem and consider a point-vortex pair embedded in a background rotation discretized with 4,8 and 16 contours. The aim here is to determine whether the findings of the previous sections are strongly dependent on the number of background contours.

In the presence of the point-vortex pair, the $N$ background contours are obviously not simply zonal, and their equilibrium positions are in fact unknowns of the problem. However, we choose the average latitude $z_{k}$ of the $k$ th contour to be equal to the expression for $z_{0 k}$ given in (6), so that the vorticity jump across that contour obeys (7). This choice is made so that the background contours, although non-zonal, be representative of solid-body rotation. 


\begin{tabular}{|c|c|c|c|c|c|c|c|c|c|c|}
\hline \multicolumn{11}{|c|}{ Equatorial vortex pairs } \\
\hline G & -1.0 & -0.15 & -0.12 & -0.1 & -0.08 & -0.05 & -0.03 & -0.01 & 0.025 & 1.0 \\
\hline$N=2$ & S & $\mathrm{S}$ & $\mathrm{O}$ & $\mathrm{O}$ & $\mathrm{O}$ & $\mathrm{O}$ & $\mathrm{O}$ & $\mathrm{T}$ & S & S \\
\hline$N=4$ & $\mathrm{~S}$ & $\mathrm{~S}$ & $\mathrm{O}$ & $\mathrm{O}$ & $\mathrm{O}$ & $\mathrm{O}$ & $\mathrm{O}$ & $\mathrm{T}$ & $\mathrm{S}$ & $\mathrm{S}$ \\
\hline$N=8$ & $\mathrm{~S}$ & $\mathrm{~S}$ & $\mathrm{O}$ & $\mathrm{O}$ & $\mathrm{O}$ & $\mathrm{O}$ & $\mathrm{O}$ & $\mathrm{T}$ & $\mathrm{S}$ & $\mathrm{S}$ \\
\hline \multicolumn{11}{|c|}{ Midlatitude vortex pairs } \\
\hline G & -1.0 & -0.15 & -0.12 & -0.1 & -0.08 & -0.05 & -0.03 & -0.01 & 0.025 & 1.0 \\
\hline$N=2$ & $\mathrm{~S}$ & $\mathrm{~S}$ & $\mathrm{~S}$ & $\mathrm{~S}$ & $\mathrm{O}$ & $\mathrm{O}$ & $\mathrm{O}$ & $\mathrm{T}$ & $\mathrm{S}$ & $\mathrm{S}$ \\
\hline$N=4$ & $\mathrm{~S}$ & $\mathrm{~S}$ & $\mathrm{~S}$ & $\mathrm{~S}$ & $\mathrm{O}$ & $\mathrm{O}$ & $\mathrm{O}$ & $\mathrm{T}$ & $\mathrm{S}$ & $\mathrm{S}$ \\
\hline$N=8$ & $\mathrm{~S}$ & $\mathrm{~S}$ & $\mathrm{~S}$ & $\mathrm{~S}$ & $\mathrm{O}$ & $\mathrm{O}$ & $\mathrm{O}$ & $\mathrm{T}$ & $\mathrm{S}$ & $\mathrm{S}$ \\
\hline
\end{tabular}

TABLE 1. The stability results for the multi-contour point-vortex pair solutions. The letters $\mathrm{S}, \mathrm{O}$ and $\mathrm{T}$ indicate stability, oscillatory instability and tearing instability, respectively. Notice that the boundaries separating the different stability regions are independent of the number of contours $N$.

In figures $12(a)$ and $12(b)$ and we show, respectively, the equatorial and midlatitude multi-contour vortex-pair equilibrium solution for $G=-1.0, d=0.2$ and $N=2,4,8$ and 16. These were obtained with a modified version of the algorithm used in $\S 5.2$ (see DiBattista 1997). Notice that the majority of the background contours are very nearly zonal, except those that are immediately next to the vortices, indicating the presence of a trapped-fluid region (cf. figure $2 \mathrm{c}$ ). We note in passing that the trapped-fluid region is present for all values of $G$, increasing very slightly in size to its maximum as $G \rightarrow \pm \infty$. We have obtained similar solutions for many values of $G$.

As in the previous section, the stability of these multi-contour vortex-pair solutions was tested with initial-value problems. Surprisingly, we have found that the stability results are virtually independent of the number of contours $N$. This is summarized in table 1 . As the number of contours is increased, no new types of instability are observed beyond those described in the previous section. Moreover, the boundaries of the stability regions appear to be very insensitive to the number of background contours.

Even the actual evolution of unstable pairs was found to be insensitive to $N$, especially for oscillatory pairs. An example is provided in figure 13, where the unstable solution for $G=-0.05$ and $N=8$ is shown. This figure should be contrasted with figure 10, showing the same case for $N=2$; the similarity is remarkable. The main difference as $N$ is increased can be seen in the long-term evolution of the more unstable cases. For instance, the tearing instability at $G=-0.01$ for the $N=8$ case, shown in figure 14, deviates from the corresponding case with $N=2$ (see figure 11) after about four days. In the eight-contour case, the tearing apart of the vortex pair occurs mainly in the meridional direction (see the last frame of figure 14), in contrast with a much more zonal separation in the two-contour case.

\section{Finite-area vortex pairs}

To increase the realism of the solutions we consider, in this last section, the desingularized analogues of the point-vortex pairs presented above; we simply replace the singular point vortices by patches of uniform vorticity and determine both the equilibrium shapes of these vortices and their stability. Such solutions (often termed 'V-states') have been obtained for many vorticity configurations in the plane and on the sphere since the original paper of Deem \& Zabusky (1978). Specifically relating 

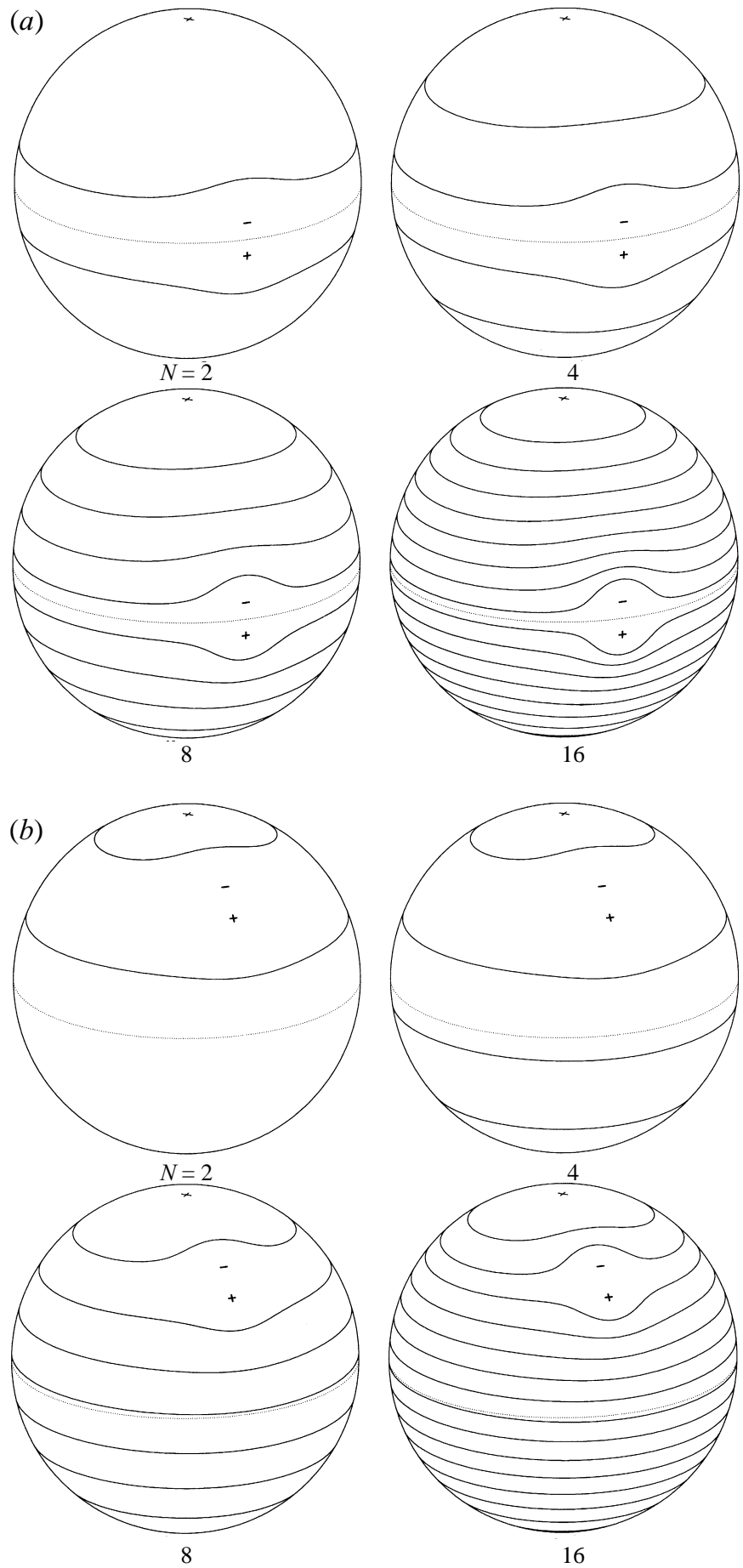

FiguRE 12. (a) Nonlinear equatorial equilibrium solutions for the westward-travelling point-vortex pair with $G=-1.0$ in multi-contour backgrounds composed of $N=2,4,8$ and 16 contours. (b) As in $(a)$, but for the midlatitude case. 

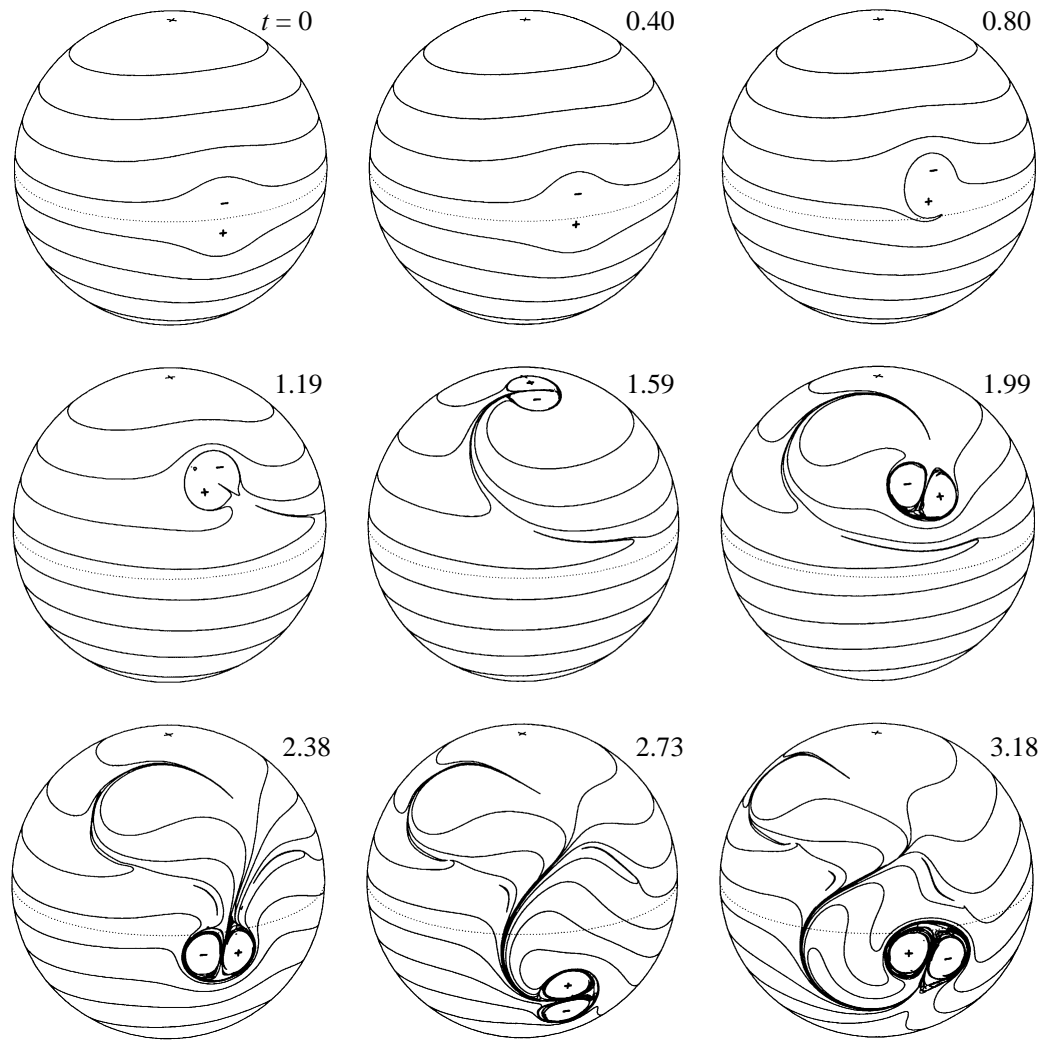

FIGURE 13. As in figure 10, but with $N=8$ background contours.

to vortex pairs, planar finite-area vortex-pair equilibrium solutions were obtained by Pierrehumbert (1980), and later completed by Saffman \& Tanveer (1982). We present here first the spherical analogues of the Pierrehumbert solutions, and then generalize these solutions to allow for the presence of a background in approximate solid-body rotation.

\subsection{In the absence of solid-body rotation}

In addition to the two geometrical parameters $d$ (the distance between the centre of vorticity of the patches) and $\lambda$ (the average latitude of the centres of vorticity) that are analogous to the ones used to define point-vortex pairs (see figure 1), the finite-area solutions require extra parameters specifying the area of the vortex patches. In principle one could specify different areas for the positive and negative vortices. To reduce the computational effort and minimize the parameter space, we limit ourselves here to determining solutions where the positive and negative vortices have equal area $A$.

In figures $15(a)$ and $15(b)$ we present spherical finite-area vortex-pair solutions for the equatorial $\left(\lambda=0^{\circ}\right)$ and midlatitude $\left(\lambda=45^{\circ}\right)$ cases, respectively. For several values of $d$ we have determined families of solutions of increasing area $A$. Like their planar counterparts, the solutions become progressively more elongated as $A$ increases, and eventually terminate when the two vortices touch. Details of the algorithm used to obtain these solutions can be found in DiBattista (1997). The solid and dotted lines 

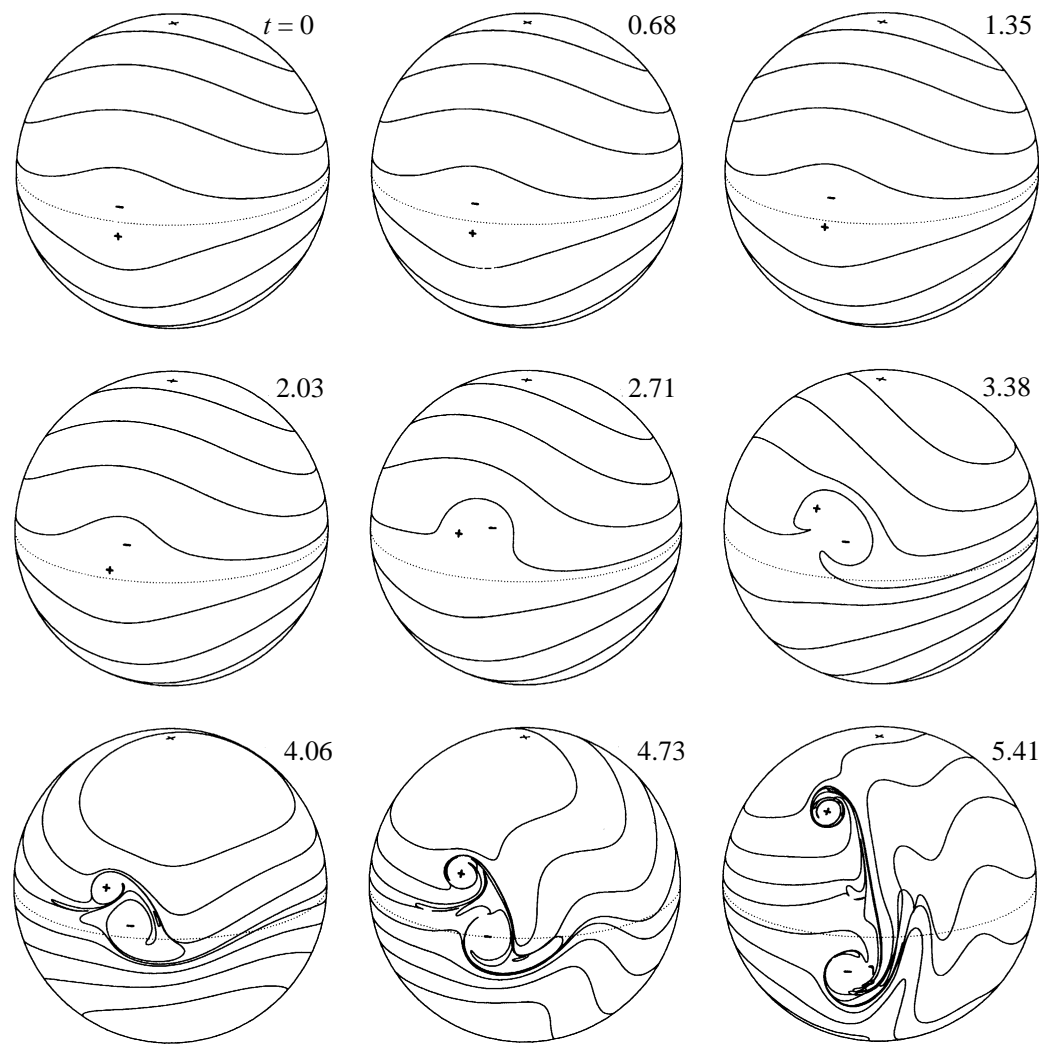

FIGURE 14. As in figure 11, but with $N=8$ background contours.

indicate the stability of the solutions, which was tested via initial-value problems as above.

As is common for most vortex-patch equilibrium solutions, an instability appears when the vortex areas are greater than a critical value (Dritschel 1985). This can be seen from the line style in figure 15; the dotted curves designate the unstable solutions, while the solid lines designate stable ones. In the midlatitude case (figure $15 b$ ) there is no symmetry between the positive and negative vortex (even though they have equal area) and our algorithm ceases to converge even when the solutions are relatively far from the case when the vortices touch. However, it does go far enough in $A$ to allow us to determine the stability boundary. For fixed $d$, equatorial pairs become unstable at a lower $A$ than do midlatitude pairs; hence, in some sense, one can say that vortex pairs are most unstable at the equator.

The instability of these finite-area pairs has no analogue in the point-vortex case; it is a novel feature that follows from the desingularization of the point vortices. We show one representative example of this 'finite-area' instability in figure 16. The key feature is that the instability proceeds by rearranging the vorticity so as to produce a vortex pair with smaller area, which is thus stable; this is accomplished by the shedding of a small vortex behind the pair (see the last frame of figure 16). This behaviour is generic to all the unstable cases we have tested in figure 15, and has also been observed for vortex pairs on the plane (Dritschel 1995). 
(a)
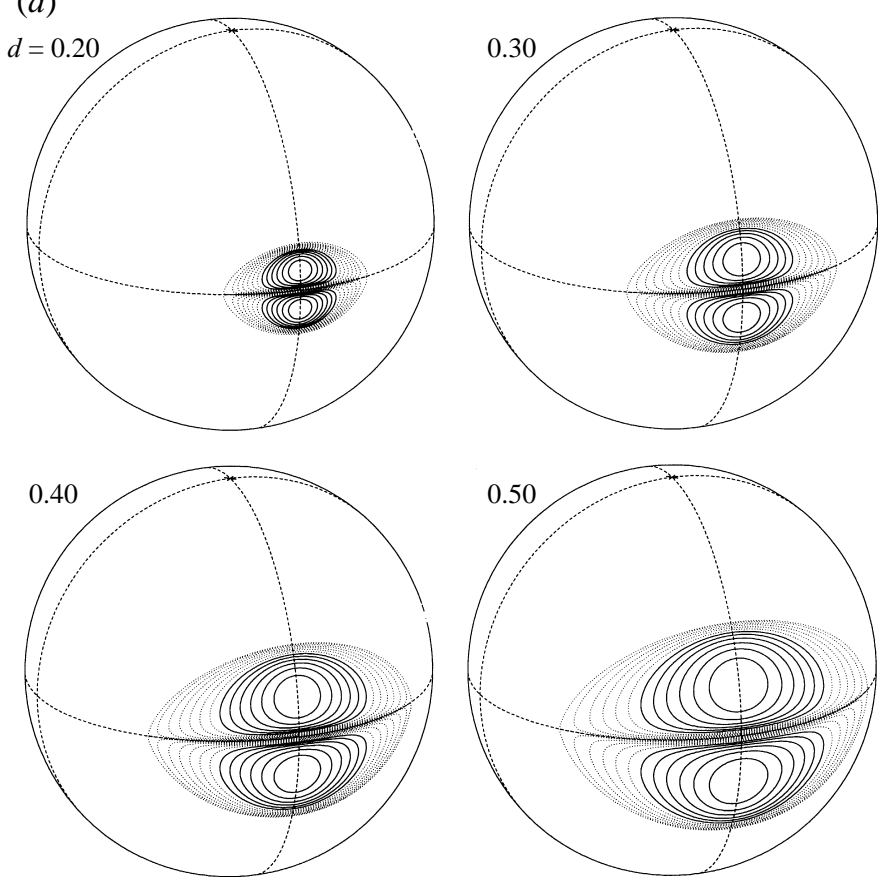

(b)
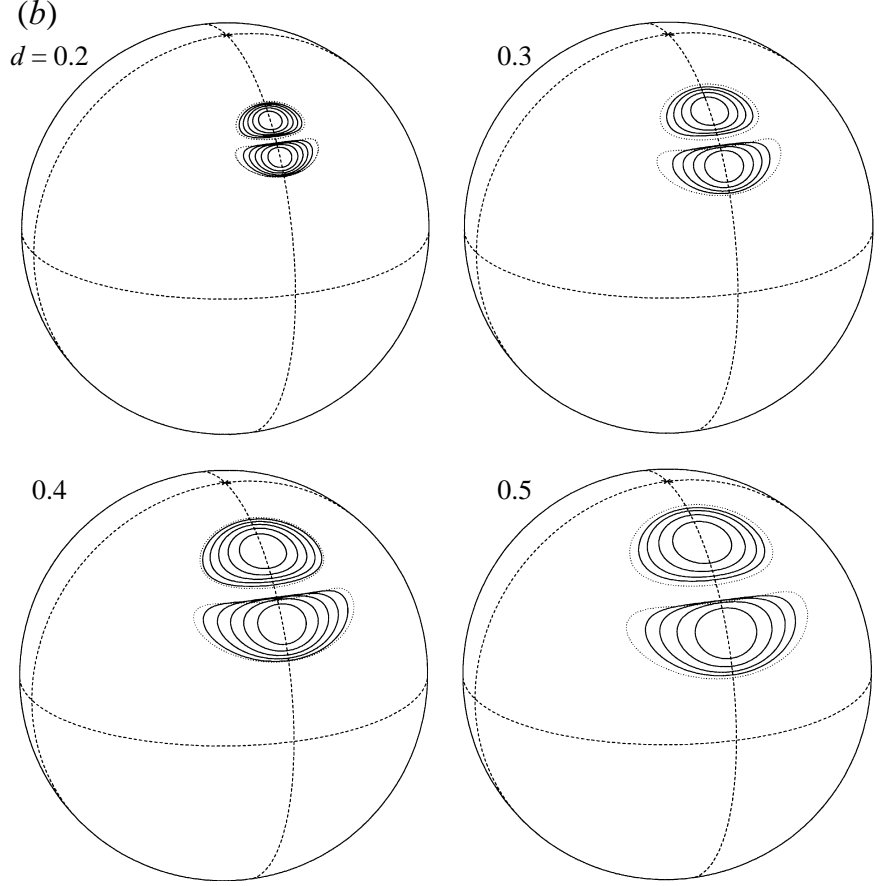

FiguRE 15. Finite-area equilibrium-solution families for $(a)$ equatorial and $(b)$ midlatitude vortex pairs on the sphere in a uniform-vorticity background, for four different values of the distance $d$ between the centres of vorticity. Solid (dotted) lines indicate stable (unstable) solutions. 

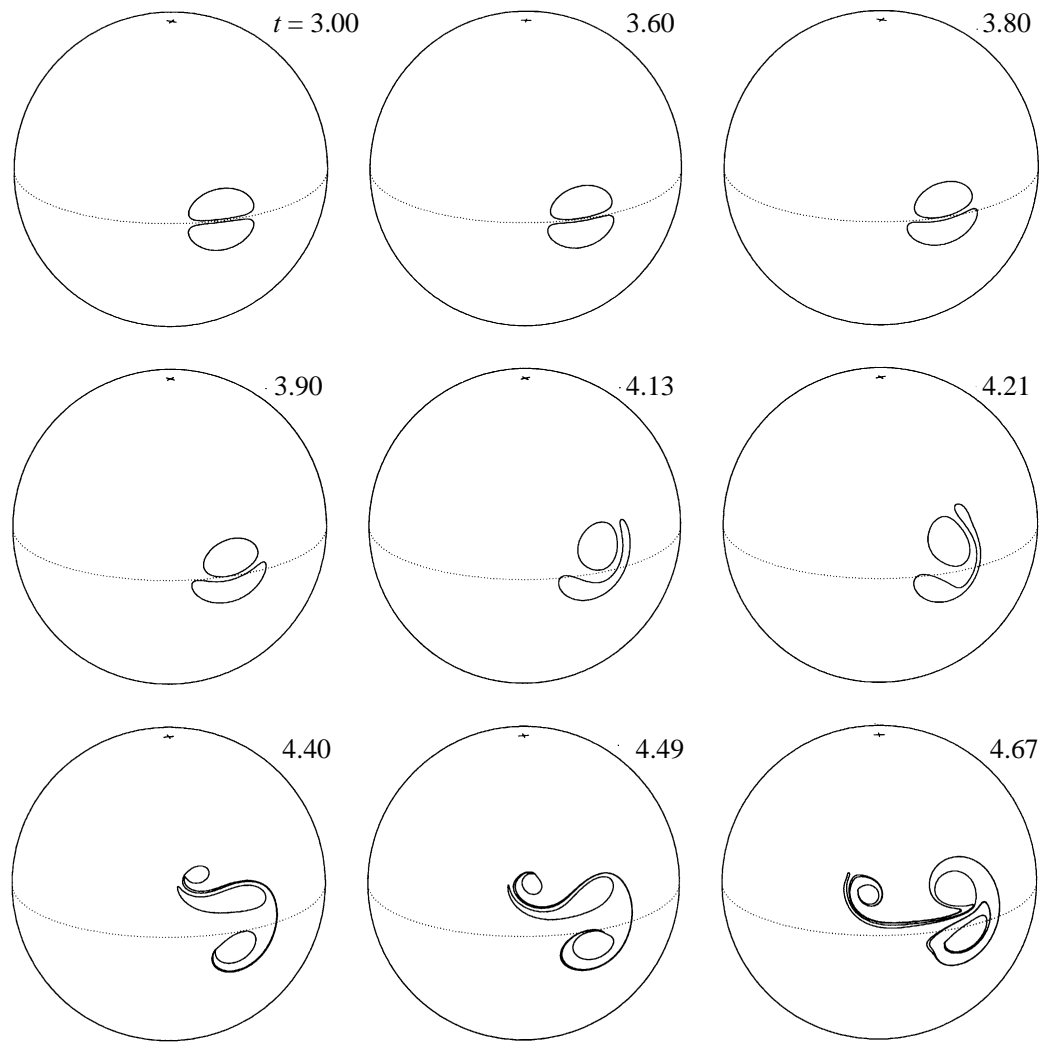

FIgURE 16. The nonlinear evolution of a slightly perturbed unstable finite-area vortex pair. The parameters of this unstable equilibrium solution are: $d=0.1$ and $A=0.07$.

\subsection{In the presence of solid-body rotation}

In this section, we construct the final and most elaborate set of solutions for vortex pairs on the sphere by embedding the above finite-area pairs in a solid-body rotation represented by $N$ contours, as in $\S 4$. For a number of representative values of $G$, these solutions are presented in figures $17(a)$ and $17(b)$ with $N=8$ for the equatorial and midlatitude cases, respectively; these solutions were obtained with one more variation of the algorithm used in previous sections. As in $\S 6$, the vorticity strips enclose equal areas and, as in $\S 7.1$, the positive and negative vortices are of equal size.

These solutions should be contrasted with the ones in figures $8(a)$ and $8(b)$ for the much simpler case of two background contours and two point vortices. The multicontour finite-area solutions are much more computationally expensive to obtain and it is more difficult to get the algorithm to converge, but they do not differ in any major way from their simpler counterparts; basically, as the area of the vortices increase the background contours become more deformed. The midlatitude solutions (figure $17 b$ ) are particularly difficult to obtain; we were unable to find solutions for $G=-0.01$ and thus have shown the closest value of $G=-0.03$ for which our scheme converges. When the vortices are strong compared to the solid-body rotation (i.e. for large $G$ ) the lower vortex becomes more elongated as the area increases, consistent with the solutions in figure $15(b)$. However, for small $G$ the situation reverses and it is the upper vortex that elongates more as $A$ increases.

The solutions in figure 17 are both finite-area and embedded in a solid-body 
Barotropic vortex pairs on a rotating sphere
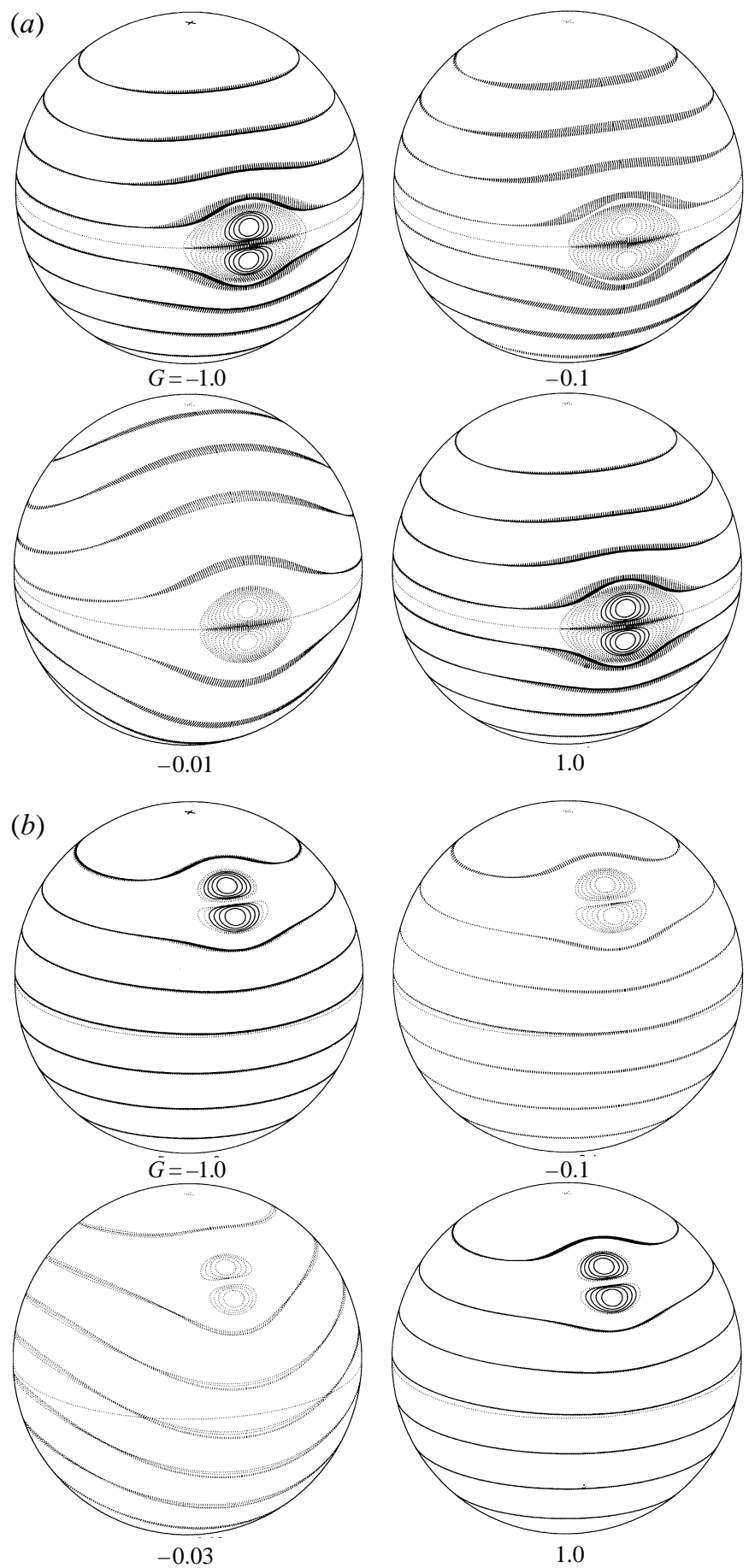

FIGURE 17. Finite-area equilibrium-solution families for $(a)$ equatorial and $(b)$ midlatitude vortex pairs embedded in a background solid-body rotation represented with $N=8$ contours. Solid (dotted) lines indicate stable (unstable) solutions. 

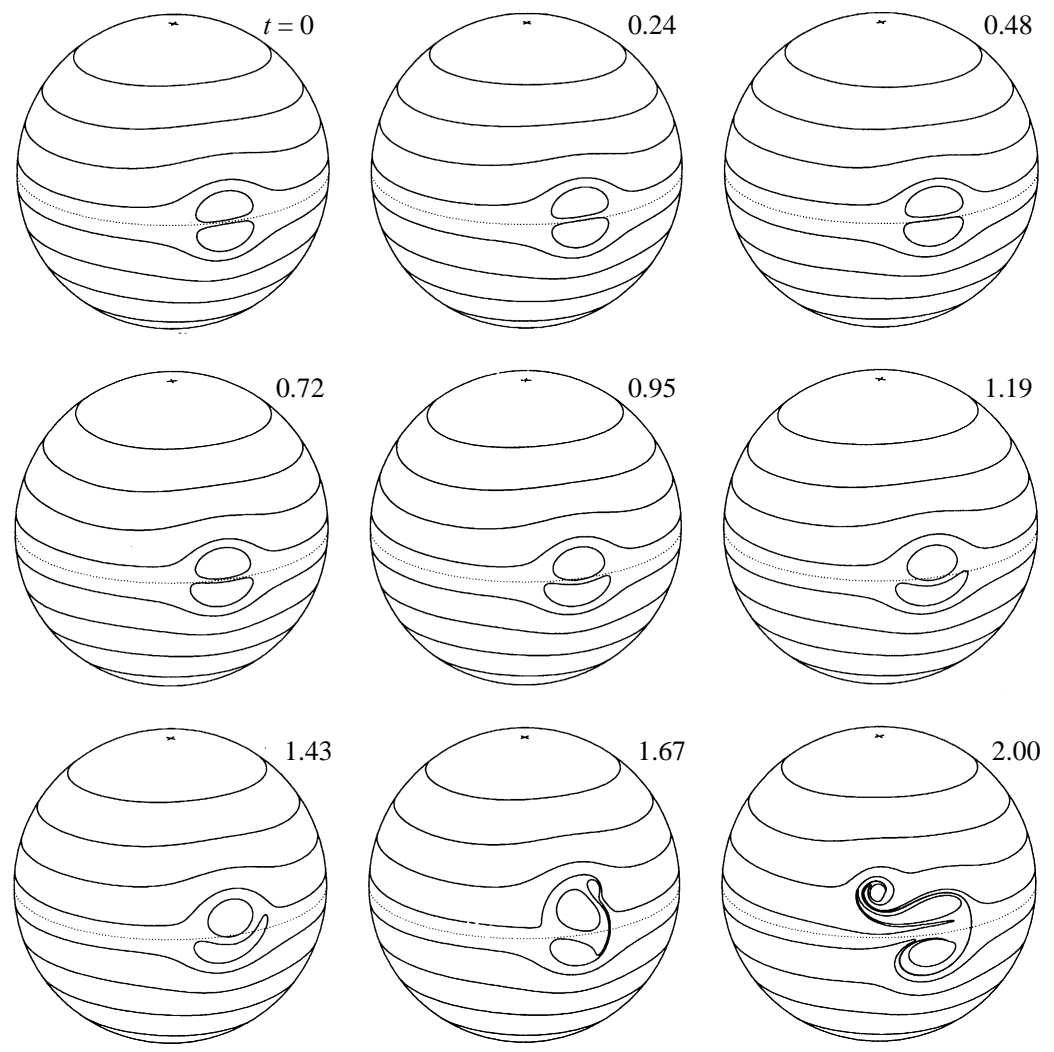

FIGURE 18. The nonlinear evolution of a slightly perturbed unstable finite-area vortex pair in a background solid-body rotation. The parameters of this unstable equilibrium solution are: $G=0.05$ and $A=0.06$. In the absence of the background flow (i.e. for $|G| \rightarrow \infty$ ) the vortex pair is stable. Notice that this is an eastward-travelling unstable solution.

rotation: it is then plausible that both point-vortex (cf. § 5.3) and finite-area (cf. § 7.1) instabilities appear in this case. In fact, we have found that the stability behaviour of these solutions is simply a combination of the finite-area and point-vortex instabilities already discussed.

First, the solid-body rotation destabilizes the corresponding finite-area solutions in the absence of a background flow. An example is given in figure 18, where an eastward-travelling pair with $G=0.05$ and $A=0.06$ is slightly perturbed and allowed to evolve. In the absence of solid-body rotation $(|G| \rightarrow \infty)$, this set of parameters yields a stable solution; the background, however, induces an instability of the finite-area type, whereby vorticity is shed from the one vortex resulting in a smaller, more stable pair. Secondly, finite-area multi-contour solutions for values of $G$ where point-vortex instabilities exist (either oscillating or tearing) are not made stable by the solid-body rotation.

More generally, the behaviour of these finite-area multi-contour solutions is a combination of finite-area and point-vortex behaviours. Representative examples are found in figure 19, showing a combination of oscillating and finite-area instabilities, and figure 20, showing a combination of tearing and finite-area instabilities. These two figures should be compared with figures 13 and 14, which exhibit only the pointvortex instabilities. The large-scale features are very similar, and the finite-area nature of the vortices only adds more complexity to the flow. 

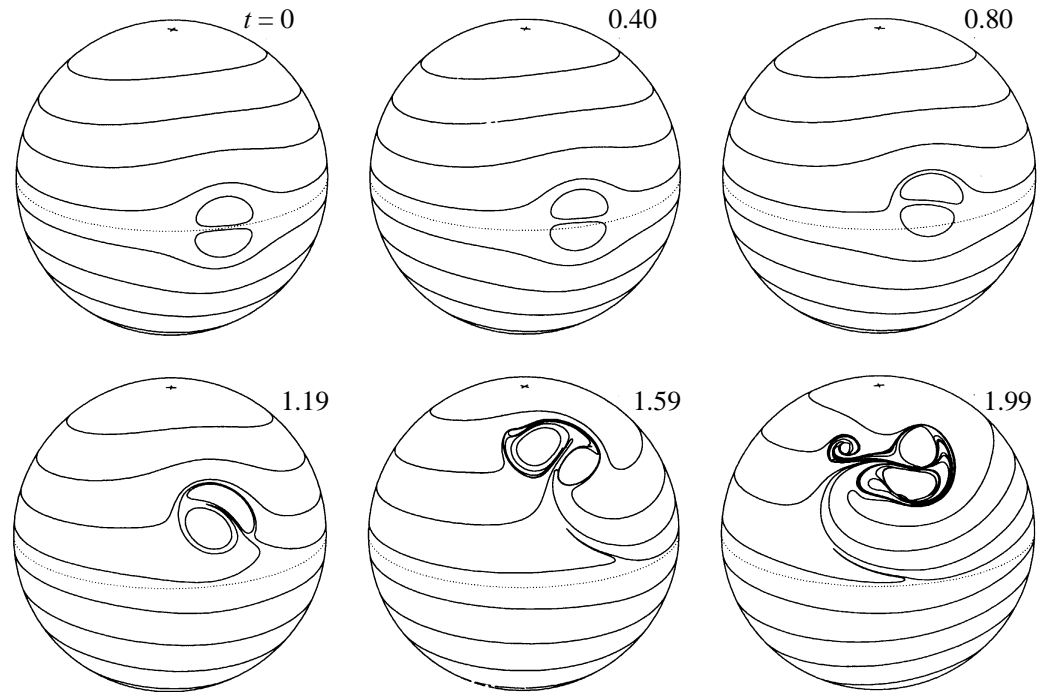

FIGURE 19. As in figure 18, but for an unstable oscillating case with $G=-0.05$ and $A=0.06$. Compare this with the evolution of the corresponding point-vortex solution in figure 13 .
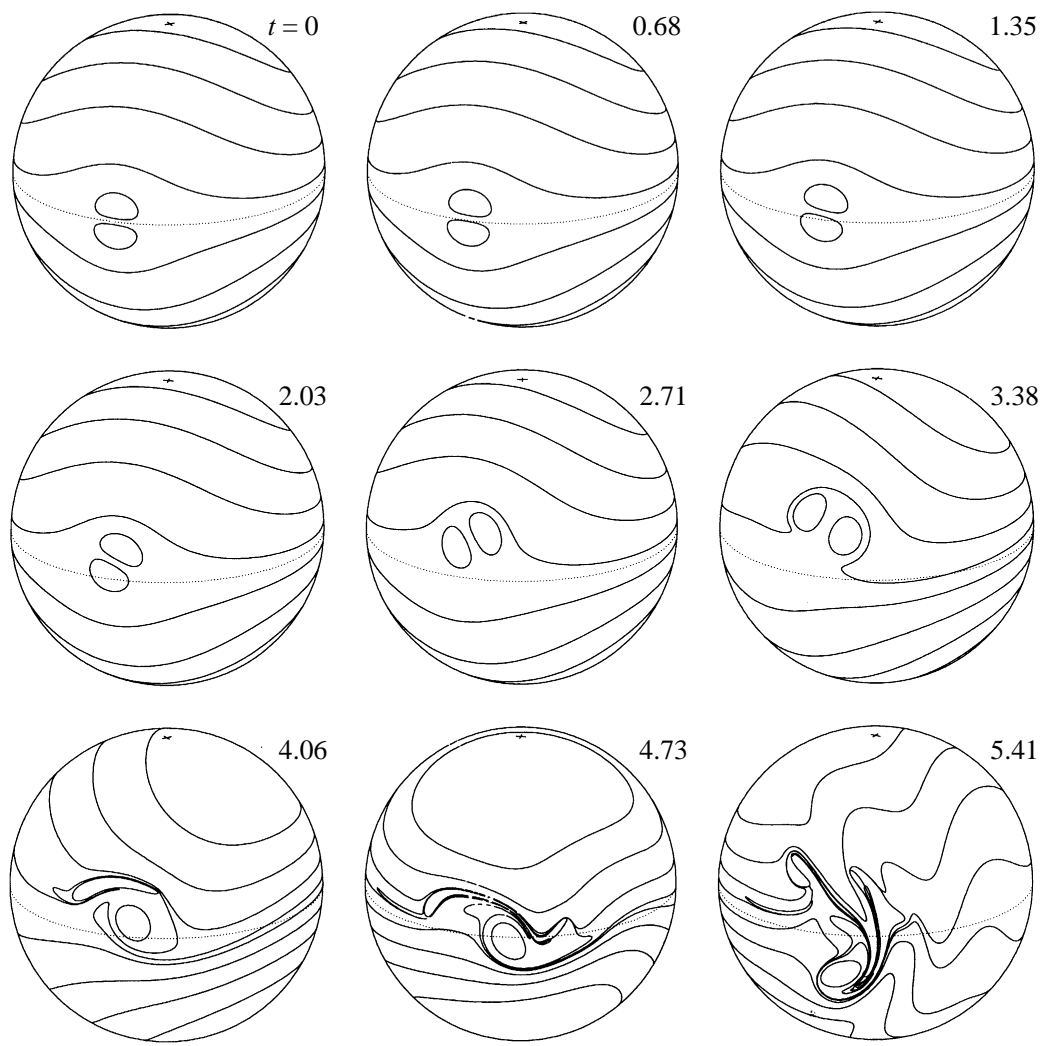

Figure 20. As in figure 18, but for an unstable tearing case with $G=-0.01$ and $A=0.04$. Compare this with the evolution of the corresponding point-vortex solution in figure 14 . 
A global view of the stability results for these finite-area multi-contour solutions can be obtained from figure 17, where solid (dotted) lines are used to show stable (unstable) solutions. It is easy to see that stable solutions exist only if (i) $G$ is either positive or sufficiently negative - otherwise a point-vortex instability occurs, and (ii) the vortex areas are not too large - otherwise finite-area instabilities are present.

\section{Discussion}

The main objective of this study has been to understand under what conditions it is possible to construct steady and stable solutions for vortex pairs within a background flow possessing a vorticity gradient. We have constructed steady vortex-pair solutions of increasing complexity, from simple point vortices surrounded by only two contours to finite-area pairs in a multi-contour background.

While it is possible to construct steady solutions for almost any parametric regime, the question of stability is really the crucial one. We have found that the critical parameter for stability is the ratio $G$ of the strengths of the vortex pair to the background flow, and we have carefully explored how the nonlinear evolution of initially perturbed steady solutions depends on this parameter.

Strong westward (approximately $G<-0.1)$ and all eastward $(G>0)$ pairs are stable, unless the the vortex sizes are too large (in which case finite-area instabilities occur). An oscillatory instability sets in as westward pairs become weak (approximately $-0.1<G<-0.01$ ); the vortex pairs wander all over the sphere (see figures 13 and 19 for typical examples). Finally, very weak westward pairs are destroyed by a tearing instability (see e.g. figures 14 and 20).

Though some of these behaviours have been previously observed on the $\beta$-plane, our study is the first to systematically explore the stability behaviour on the sphere. In particular, the stability of strong westward pairs and the onset of instability as pairs become weak are novel results which cannot be obtained by $\beta$-plane studies, because they are crucially dependent on the curvature of the sphere.

As for the original question of whether or not the instability of the modon solution is an exceptional or generic property of vortex pairs in rotating flows, we argue that it is probably generic since it can be isolated in the simplest vortex-pair model (cf. $\S 5)$ and it persists as the model is made more complex. The resonance with Rossby waves appears very clearly in the simplest linear model and adding complexity, as we have shown, does not remove this fundamental mechanism for instability. In fact, more complexity introduces futher modes of instability.

Finally, one may ask to what degree these results may be applied to the understanding of atmospheric blocking events. The solutions of interest to atmospheric blocking fall in the weak westward regime (McWilliams 1980). In that regime, all of the steady solutions presented in this paper (independent of their complexity), as well as the modon solutions (Nycander 1992), are known to be unstable. This fundamental instability makes it difficult to argue that blocking events somehow correspond to unforced barotropic solutions of the sort we have presented here. Hence, the results of this paper suggest that the robustness and persistence of blocking events must be attributed to not yet fully understood forcing mechanisms, as has been discussed elsewhere (Shutts 1983, 1986; Nakamura, Nakamura \& Anderson 1997).

The computations herein were performed at the Pittsburgh Supercomputing Center. This work is supported by the United States National Science Foundation, and by the DOE through a Computational Science Fellowship to MTD. 


\section{REFERENCES}

BAINES, P. G. 1976 The stability of planetary waves on a sphere. J. Fluid Mech. 73, 193-213.

Batchelor, G. K. 1967 Introduction to Fluid Dynamics. Cambridge University Press.

DeEm, G. S. \& Zabusky, N. J. 1978 Vortex waves: Stationary 'V-states', interactions, recurrence, and breaking. Phys. Rev. Lett. 40, 859-862.

DiBattista, M. 1997 Vortex pairs on the rotating sphere. PhD thesis, Columbia University.

Dritschel, D. G. 1985 The stability and energetics of corotating uniform vortices. J. Fluid Mech. 157, 95-134.

DrITSCHEL, D. G. 1989 Contour dynamics and contour surgery: numerical algorithms for extended high-resolution modeling of vortex dynamics in two-dimensional, inviscid, incompressible flows. Comput. Phys. Rep. 10, 77-146.

Dritschel, D. G. 1995 A general theory for two-dimensional vortex interactions. J. Fluid Mech. 293, 269-303.

Dritschel, D. G. \& Polvani, L. M. 1992 The roll-up of vorticity strips on the surface of a sphere. J. Fluid Mech. 234, 47-69 (referred to herein as DP92).

Flierl, G. R., Larichev, V. D., McWilliams, J. C. \& Reznik, G. N. 1980 The dynamics of baroclinic and baroclinic eddies. Dyn. Atmos. Oceans 5, 1-41.

Haurwitz, B. 1940 The motion of atmospheric disturbances on the spherical earth. J. Mar. Res. 3, 254-267.

Hobson, D. D. 1991 A point vortex dipole model of an isolated modon. Phys. Fluids A 3, 3027-3033.

Kimura, Y. \& Oкамото, H. 1987 Vortex motion on a sphere. J. Phys. Soc. Japan 56, 4203-4206.

LAMB, H. 1932 Hydrodynamics. Dover.

Larichev, V. D. \& Reznik, G. M. 1976 Two-dimensional Rossby soliton: an exact solution. POLYMODE News 19, 3 and 6.

Matsuoka, C. \& Nozaki, K. 1992 Point vortex model of a modon solution. Phys. Fluids B 4, $551-558$

McWilliams, J. C. 1980 An application of equivalent modons to atmospheric blocking. Dyn. Atmos. Oceans 5, 43-66.

McWilliams, J. C., Flierl, G. R., Larichev, V. D. \& Reznik, G. N. 1981 Numerical studies of barotropic modons. Dyn. Atmos. Oceans 5, 219-238.

Nakamura, H., Nakamura, M. \& Anderson, J. L. 1997 The role of high- and low-frequency dynamics in blocking formation. Mon. Weather Rev. 125, 2074-2093.

Nycander, J. 1992 Refutation of stability proofs for dipole vortices. Phys. Fluids A 4, 467-476.

Pierrehumbert, R. T. 1980 A family of steadily translating vortex pairs with distributed vorticity. J. Fluid. Mech. 99, 129-144.

Polvani, L. M. \& Dritschel, D. G. 1993 Wave and vortex dynamics on the surface of a sphere. $J$. Fluid Mech. 255, 35-64 (referred to herein as PD93).

Saffman, P. G. \& Tanveer, S. 1982 A touching pair of equal and opposite uniform vortices. Phys. Fluids 25, 1929-1930.

SHutTs, G. J. 1983 The propagation of eddies in diffluent jetstreams: Eddy vorticity forcing of blocking flow fields. Q. J. R. Met. Soc. 109, 737-761.

ShutTs, G. J. 1986 A case study of the eddy forcing during an Atlantic blocking episode. Adv. Geophys. 29, 135-162.

Stern, M. E. 1975 Minimal properties of planetary eddies. J. Mar. Res. 33, 1-13.

Sutyrin, G. G., Hesthaven, J. S., Lynov, J. P. \& Rasmussen, J. J. 1994 Dynamical properties of vortical structures on the $\beta$-plane. J. Fluid Mech. 268, 103-131.

Tribbia, J. J. 1984 Modons in spherical geometry. Geophys. Astrophys. Fluid Dyn. 30, 131-168.

Verkley, W. T. M. 1984 The construction of barotropic modons on the sphere. J. Atmos. Sci. 41, 2492-2504.

VERKLeY, W. T. M. 1987 Stationary barotropic modons in westerly background flows. J. Atmos. Sci. 44, 2383-2398.

Zabusky, N. J. \& McWilliams, J. C. 1982 A modulated point vortex model for geophysical $\beta$-plane dynamics. Phys. Fluids A 25, 2175-2182. 